\title{
Determination of Actual Object Size Distribution from Direct Imaging
}

\author{
Md. Iqbal Hossain, Tao Chen, Yanhui Yang, and Raymond Lau* \\ School of Chemical and Biomedical Engineering \\ Nanyang Technological University \\ 62 Nanyang Drive, Singapore 637459
}

\begin{abstract}
Direct imaging is a technique commonly used in the study of particle, bubble, and droplet size distribution in a dynamic system. Objects such as particles, bubbles, and droplets can be present at various distances from the imaging device when images are captured. Hence, the location of the object will need to be known in order to determine the actual size of an individual object. However, the location of the object cannot be determined from a single image. A single calibration scale defined at the focusing plane is normally used for the determination of all the object sizes from images. When the focus is close to the imaging device, the change in magnification with location is large. The size distribution obtained from the use of a single calibration scale would thus give a considerable deviation from the actual size distribution. In this study, a statistical method is proposed to reconstruct the actual object size distribution from the experimental object size distribution obtained from images using a single calibration scale defined at the focusing plane. Experiments are performed to validate the accuracy of the proposed method on the particle size distribution determination in a settling system. The stability of the proposed method is also analyzed theoretically for imaging devices with different depth-of-field (DOF), focusing location, and change in magnification with distance.
\end{abstract}

\section{Introduction}

The size distribution of the discrete phases in multiphase systems such as the size distribution of particles in gas-solid or liquid-solid systems, bubbles in gas-liquid or gas-liquid-solid systems, and droplets in liquid-liquid systems is important for the operation of the specific applications. The knowledge of size distribution can enhance the understanding of mixing, heat and mass transfer properties for design and scale-up of the operations. It can also be used for validation or as a parameter in process simulations using computational fluid dynamics (CFD). However, the measurement of size distribution with reasonable accuracy has always been a challenge. Numerous techniques including Doppler anemometry techniques, tomographic techniques, invasive probe techniques, and direct imaging have been developed to measure the size distribution of the discrete phases. ${ }^{1-3}$ Doppler anemometry techniques such as phase Doppler anemometry (PDA) can give a measurement of size but limited to the spherical objects at low volumetric fractions of the discrete phases. Tomographic measurements reconstruct the images of the distinct phases from the data obtained by the sensors using a reconstruction algorithm. However, tomographic techniques normally have limited spatial resolution and there is a lack of reliable image reconstruction algorithm. A tomographic system employing wire-mesh sensors can give a measurement of size without the use of any image reconstruction. ${ }^{4}$ The problem is that the system becomes highly invasive and it is limited to a certain type of continuous phases. ${ }^{2,4}$ Moreover, complications arise at the

To whom correspondence should be addressed

Email address: wmlau@ntu.edu.sg

Telephone: $+6563168830 \quad$ Fax: +6567947553 
conditions of high volumetric fractions of the discrete phases. Invasive probe techniques such as focused beam reflectance measurement (FBRM), optical fiber probe, conductivity probe, etc give only the cord-length distribution. The cord-length distribution needs to be converted into the size distribution which is another challenging area. Direct imaging is preferred over other techniques to give a direct measurement of the size and shape of the discrete phases.

One limitation with the use of direct imaging for size determination is that the size of the object image varies according to the horizontal distance of the object from the imaging device. As illustrated in Figure 1, the larger the distance between the object and the imaging device, the smaller the size of the object image and hence, the smaller the magnification (the ratio of the object size obtained from image to the actual size of the object). However, it is difficult to determine the distance between the object and the imaging device from the image itself and a single calibration scale is normally used for the object images. Hence, the size measurement from direct imaging is prone to errors as a result of the uncertainty in the calibration scales to be used. This problem is especially important for imaging devices that yield large difference in magnifications with the distance between the object and the imaging device, such as borescope and macroscopic image measurements. One common approach is to reduce the depth-of-field (DOF) (viewable distance) and consider only 'in-focus' objects. The change in magnification within the reduced DOF is then considered negligible. ${ }^{5-10}$ However, a reduction in DOF may not always be possible and the sampling time will be substantially increased with a reduced DOF. In this study, it is proposed to take the difference in magnification within the DOF into consideration and use a statistical method to determine the actual object size distribution from direct imaging.

\section{Methodology}

Magnification Model. The magnification of an object at different horizontal distance within the DOF is an important parameter for the reconstruction of the actual size distribution. The magnification in this study is defined as the ratio of the object size obtained from image to the actual size of the object. An object located at the focusing plane will have a magnification of 1 and the object size obtained from image will be the same as the actual object size.

As illustrated in Figure 2, consider an object of actual size $R$ located at a distance $z_{1}$ away from a lens of focal length $f$. The well focused image of this object is created at a distance $v_{1}$ away on the other side of the lens. The relationship between the focal distance, $f$, image distance $v_{l}$ and the object distance $z_{1}$ can be described by the following lens equation: ${ }^{11}$

$$
\frac{1}{z_{1}}+\frac{1}{v_{1}}=\frac{1}{f}
$$

If the same object is moved to another position which is $z_{2}$ distance away from the lens, the well focused image will be created at a distance $v_{2}$. The relationship between $f, z_{2}$ and $v_{2}$ would be the same as eq 1. It is assumed that $y_{w .1}$ and $y_{w .2}$ are the size of the well focused images created at $v_{1}$ and $v_{2}$ respectively. $y_{w .1}$ and $y_{w .2}$ can then be related to each other by the following equation:

$$
\frac{y_{w .2}}{y_{w .1}}=\frac{1+\frac{z_{1}}{k_{1}}}{1+\frac{z_{2}}{k_{1}}}
$$

where $k_{l}$ is a function of the focal length only. However, most of the imaging device consists of multiple lenses. In a multiple lens system, the relationship between the image sizes $y_{w . l}$ and 
$y_{w .2}$ would still follow eq 2 while $k_{l}$ becomes a function of the focal length of each lens, the number of lens as well as the lens separation distance etc. For a direct image capturing system, the position of the sensor/film is usually fixed. Therefore, the sensor plane can coincide with the well focused image plane only when the object is located at the focusing plane. If the object is not on the focusing plane, the image formed at the sensor plane will appear blurry. The larger the distance the object from the focusing plane, the blurrier the image would be. Moreover, because of the blurred image, the size obtained from image no longer follows eq 2. A modification was proposed by Lee et al. ${ }^{11}$ to include the blurring effect:

$$
\frac{y_{2}}{y_{1}}=\frac{1+\frac{z_{1}}{k_{1}}}{1+\frac{z_{2}}{k_{1}}} \cdot \frac{1-\frac{z_{2}}{k_{2}}}{1-\frac{z_{1}}{k_{2}}}
$$

Consider a calibration scale being defined at the focusing plane $z_{F}$, the magnification of the image can be considered as:

$$
M^{\prime}(z)=\frac{y}{R}=\frac{1+\frac{z_{F}}{k_{1}}}{1+\frac{z}{k_{1}}} \cdot \frac{1-\frac{z}{k_{2}}}{1-\frac{z_{F}}{k_{2}}}
$$

where $y$ is the object size obtained from the image of the object located $z$ distance away from imaging device, $R$ is the actual size of the object, and $k_{1}$ and $k_{2}$ are two parameters which can be determined experimentally. Since only objects within the DOF are considered visible, the magnification is only defined when $z_{n} \leq z \leq z_{r}$, where $z_{n}$ is the near-end limit of the DOF and $z_{r}$ is the rear-end limit of the DOF.

Reconstruction of Actual Object Size Distribution. The object size obtained from image, $y$ depends on the distance between the object and the imaging device, $z$ as well as the actual object size, $R$. The relationship among $y, z$ and $R$ can be described by the magnification model in eq 4 . Theoretically, the probability density function $(p d f)$ of the object size distribution obtained from images, $p(y)$ can be described by the equation:

$$
p(y)=\int_{0}^{\infty} p(y \mid R) p(R) d R
$$

where $p(y \mid R)$ is the conditional $p d f$ of obtaining an object size $y$ from the image of the object with an actual size $R$. Typically, the size of particles or bubbles follows a log-normal distribution $p(R)$ with two parameters $\mu$ and $\sigma .^{12-15}$ These two parameters can be optimized using least squares method such that the solution of eq 5 gives a $p(y)$ that closely matches the experimental object size distribution obtained from images, $p_{e}(y)$. However, a general analytical solution to eq 5 does not exist, although for some special case this is possible. ${ }^{16-18}$ A numerical procedure is then adopted to approximate the integral in eq 5 . This approximation would require the size range of the actual object size distribution $p(R)$. Since $p(R)$ is unknown, a size range wide enough to cover the entire $p(R)$ will need to be assumed. One approach is to assume that the size range of $p(R)$ is the same as that of $p_{e}(y)$. If the sample size is large, due to the magnifications involved at different object locations, $p_{e}(y)$ will indeed cover a wider size range than the $p(R) . M$ number of bins with bin size $\Delta R$ and mid-point sizes $R_{j=1, \ldots, M}$ can be generated within the assumed size range of $p(R)$. The object size distribution obtained from images can then be approximated by: 


$$
p(y) \approx \sum_{j=1}^{M} p\left(y \mid R_{j}\right) p\left(R_{j}\right) \Delta R
$$

$p\left(y \mid R_{j}\right)$ can be expanded for various horizontal distance $z$. It is reasonable to assume that objects have equal probability to appear along the viewable distance. Formally this is equivalent to

$$
p(z \mid R)=\left\{\begin{array}{lc}
k ; & z_{n} \leq z \leq z_{r} \\
0 ; & \text { otherwise }
\end{array}\right.
$$

To simplify the calculation, the viewable horizontal distance is divided into $N$ equally spaced sections where the mid-point of each section is termed as $z_{1}, z_{2}, \ldots, z_{N}$. Therefore, the location probability for each section can be given by the equation:

$$
p\left(z_{i} \mid R_{j}\right) \Delta z=\frac{1}{N}
$$

where

$$
\Delta z=\frac{z_{r}-z_{n}}{N}
$$

Hence, $p\left(y \mid R_{j}\right)$ can be approximated as

$$
p\left(y \mid R_{j}\right)=\int p\left(y \mid R_{j}, z\right) p\left(z \mid R_{j}\right) d z \approx \sum_{i=1}^{N} p\left(y \mid R_{j}, z_{i}\right) p\left(z_{i} \mid R_{j}\right) \Delta z
$$

Substituting eq 10 into eq $6, p(y)$ can be estimated as:

$$
p(y) \approx \sum_{j=1}^{M}\left[\sum_{i=1}^{N} p\left(y \mid R_{j}, z_{i}\right) p\left(z_{i} \mid R_{j}\right) \Delta z\right] p\left(R_{j}\right) \Delta R
$$

$p\left(y \mid R_{j}, z_{i}\right)$ is found to be constant experimentally for each $R_{j}$. Finally, the reconstructed object size distribution, $p_{r}(R)$ can be obtained using least square method by optimizing the parameters of $p(R)$ such that the approximated $p(y)$ closely resembles $p_{e}(y)$.

It is noted that the choice of $N$ and $M$ would affect the solution accuracy and overall computational time. $N$ depends on the degree of change of magnification within the DOF. For a large degree of change in magnification within the DOF, a large $N$ is needed to obtain a smooth solution for the approximated $p(y)$. For the application of borescope used in this study, it can be considered to have a large degree of change in magnification. It is found that a minimum $N$ of 50 is needed to obtain a reasonable solution using eq 11 . The choice of $M$ will be discussed in the latter section.

Technically, $p(R)$ does not need to follow a particular distribution function. However, optimization problem arises especially when the number of bins, $M$ in $p(R)$ is large. Nonetheless, the type of probability distribution function used can still be specified depending on the system and the specific application.

\section{Experimental Section}

The methodology proposed in this study is verified with the size measurement of spherical poly vinyl chloride (PVC) particles in a dynamic system. The actual size distribution of the PVC particles used is determined by direct imaging under static condition. All the PVC particles are placed evenly on a flat horizontal surface accompanied by a known scale. A video camera (Olympus I-speed) equipped with a $28-75 \mathrm{~mm}$ lens is placed perpendicular to the horizontal surface. Enough static images are taken for different batch of particles to make sure that the sample size is larger than 600. The actual sizes of the particles are then 
determined using an in-house developed MATLAB image analysis algorithm capable of treating both well-focused and blurred images. In this algorithm, objects (the PVC particles) are identified using region segmentation based on local thresholding. ${ }^{19-21}$ As the particles are not completely spherical, the maximum dimension of the contour of a particle image is taken as the particle size.

A system of particles settling through a pool of water is chosen as the model dynamic system. The schematic of the experimental setup is shown in Figure 3. Two acrylic columns having internal diameters of 25 and $140 \mathrm{~mm}$ are used. A rigid borescope ${ }^{22-31}$ (Olympus) with a 60 field of view and $0^{\circ}$ direction of view is used to obtain particle images in a dynamic system. The borescope has a diameter of $6 \mathrm{~mm}$ and length of $250 \mathrm{~mm}$. The borescope is coupled with the video camera (Olympus I-speed) capable of recording images at $1,000 \mathrm{~Hz}$ and a resolution of $800 \times 600$. The borescope is inserted from the side of the column and the column is filled with water. The water level is kept at $20 \mathrm{~mm}$ above the borescope location to minimize particles dispersion in the borescope viewable distance during particle settling. At the start of the experiment, particles are dropped evenly with the use of a vibrating mesh. The vibrating frequency is adjusted to maintain a slow particle dropping rate so as to maintain a low particle concentration in the column during the course of settling.This is to ensure that the borescope can capture all the particles settling in the viewable distance of the borescope. The focus of the borescope is set at $5 \mathrm{~mm}$ in front of the borescope tip. A $1000 \mathrm{~W}$ external halogen light is used as the illumination source. Images of the settling of particles are recorded at a resolution of $800 \times 600$ with a shutter speed of $1 / 500$ second to minimize motion blur. For particle settling in the $25 \mathrm{~mm}$ ID column, the viewable distance of the borescope is limited by the column wall. For particle settling in the $140 \mathrm{~mm}$ ID column, a 20 $\times 40 \mathrm{~mm}$ mesh with $3.35 \mathrm{~mm}$ square orifice is used during the particle dropping process to confine the viewable distance. The radial positions of the borescope in the two columns are shown in Figure 4.

There is a certain degree of barrel distortion in the borescope image due to the wide angle design of the borescope lens. The barrel distortion is corrected by the method proposed by Haneishi et al. ${ }^{32}$ The magnification at various distances from the borescope is calibrated in water. The borescope focus is fixed at $5 \mathrm{~mm}$ from the borescope tip. The object size obtained from the image of the object located at the focusing plane is considered to be the same as the actual object size. The same calibration scale is used to determine the size of all the objects in the images regardless of the actual object locations. The magnification at a distance $z$ from the borescope tip is then defined as the ratio of the object size obtained from the image of the object located at $z$ to the actual size of the object. In this case, the magnification at the focusing plane would be 1 . This magnification also considers the blurriness of the object image when it is not located at the focusing plane. The relationship between magnification and distance from the borescope is shown in Figure 5. Eq 4 is fitted with the experimental data, and $k_{1}$ and $k_{2}$ are found to be 4.54 and 100.34 respectively. It can be seen in Figure 5 that the change in magnification is high when the object is near the borescope and the change in magnification is progressively lowered when the object is moved away from the borescope.

\section{Results and Discussion}

Experimental Verification. The DOF of the measurement is a crucial parameter in the determination of the actual size distribution. DOF depends on the blurriness of the object and it is an analog parameter. In order to demonstrate the applicability of the proposed method, 
two different setups are used to limit the DOF. As mentioned in the experimental section, the viewable distance of the borescope in the $25 \mathrm{~mm}$ ID column is limited by the column wall. With the borescope being placed $8 \mathrm{~mm}$ from the column wall, the DOF would be $16 \mathrm{~mm}$. A comparison of the experimental particle size distribution obtained from borescope images using a calibration scale at the focusing plane, $p_{e}(y)$, the reconstructed particle size distribution based on the proposed method, $p_{r}(R)$ and the actual particle size distribution, $p(R)$ of this experimental setup is shown in Figure 6. It can be seen that $p_{e}(y)$ has a larger variance and smaller average than $p(R)$. In this experimental setup, the focus of the borescope is set at $5 \mathrm{~mm}$ from the borescope tip. As shown in Figure 5, the magnification at $z<5 \mathrm{~mm}$ is greater than 1. Object appearing in this region will appear to be larger than its actual size. On the other hand, at $z>5 \mathrm{~mm}$, the magnification is smaller than 1. Object appearing in this region will appear to be smaller than its actual size. Therefore, the size range of $p_{e}(y)$ would spread across a larger range than the actual size range. It is known that the front DOF $\left(z_{F}-z_{n}\right)$ is normally shorter than the rear DOF $\left(z_{r}-z_{F}\right){ }^{33-35}$ In the total viewable distance of $1-17 \mathrm{~mm}$, the viewable distance in front of the focusing plane $(1-5 \mathrm{~mm})$ is commonly known as front DOF whereas the viewable distance beyond the focusing plane $(5-17 \mathrm{~mm})$ is known as rear DOF. As the front DOF is shorter than the rear DOF, the number of particles having lower magnification is larger than that of having higher magnification. Hence, $p_{e}(y)$ would be distributed toward the lower size range. Nonetheless, $p_{e}(y)$ is unable to give a good indication of the $p(R)$. However, once $p_{e}(y)$ is treated with the proposed method, it can be seen that $p_{r}(R)$ is in good agreement with the actual size distribution. It can be seen from Table 1 that the cumulative square error of the size distribution is improved from 5.320 with $p_{e}(y)$ to 0.183 with $p_{r}(R)$.

For particle settling in a $25 \mathrm{~mm}$ ID column, wall effect may be substantial. Therefore, particle settling in a $140 \mathrm{~mm}$ ID column is also performed. The viewable distance of the borescope in the $140 \mathrm{~mm}$ ID column is limited by a mesh of size $20 \times 40 \mathrm{~mm}$. The DOF of this setup is then limited to 1 and $21 \mathrm{~mm}$ from the borescope tip. A comparison of $p_{e}(y), p_{r}(R)$ and $p(R)$ for this experimental setup is shown in Figure 7. Similar to the previous setup, $p_{e}(y)$ has a larger variance and smaller average than $p(R) . p_{r}(R)$ is also found to be in good agreement with $p(R)$. The cumulative square error of the size distribution is improved from 5.800 with $p_{e}(y)$ to 0.089 with $p_{r}(R)$. The cumulative square error of $p_{r}(R)$ for this experimental setup is smaller than that for the previous setup. The presence of wall effect and the curvature of the inner column wall reduce the amount of particles settling near the column wall. Therefore, the actual DOF of the borescope in the $25 \mathrm{~mm}$ ID column is not exactly the same as the DOF used in the reconstruction algorithm. Hence, $p_{r}(R)$ has a larger cumulative square error in the $25 \mathrm{~mm}$ ID column than that in the $140 \mathrm{~mm}$ ID column. Nonetheless, the proposed method still gives a significant improvement to $p_{e}(y)$, especially when the magnification change within the DOF is large.

The viewable distance of the borescope used in this study is limited by the experimental setup. For normal application of the proposed technique where DOF is an optical property, the DOF of the imaging device will need to be determined separately. Through digital imaging, the object images can be treated with certain criteria to identify the edge of the objects from the images. Calibration can then be performed with object images at known distances from the imaging device. When the blurriness of the edge is beyond the identification of the digital treatment, the object can be considered outside the DOF and the limits of the DOF can be determined. A number of research works on the identification of the DOF are available in the literature that are based on similar principles. ${ }^{33-42}$ 
Theoretical Analysis. Borescope is used in two different setups to demonstrate the applicability of the proposed method under different conditions. However, the effects of other factors such as change in DOF with aperture size, the degree of change in magnification with distance from lens, etc have not been experimentally tested. Theoretical analysis is then performed to examine the effect of various factors on the accuracy of the proposed method. An evaluation of the appropriate number of bins and choice of distribution function for the reconstruction is also proposed.

In the theoretical analysis, a known log-normal size distribution $(\mu=0.9$ and $\sigma=0.2)$ is used as $p(R)$ and the size range is considered to be from 1-5 mm. It is assumed that objects with size distribution of $p(R)$ are uniformly distributed within the DOF and the object concentration is sufficiently low that the probability of having front objects blocking the rear objects is negligible. The relationship between magnification and the distance between object and lens is also assumed to be following eq 4. The overall size range of $p(R)$ is divided into $M$ number (typically 40) of equally spaced bins. The viewable horizontal distance is also discretized into $N$ equally spaced sections (typically 8 sections per $1 \mathrm{~mm}$ ). Therefore, the object size distribution obtained from images can be simulated using eq 11. A comparison of the object size distribution obtained from simulation, $p_{s}(y)$ and $p_{e}(y)$ is made for the PVC particles used in both of the setup described in the experimental section. It can be seen from Figure 8 that $p_{s}(y)$ can closely resemble $p_{e}(y)$ for both cases. In Figure $8, p_{e}(y)$ deviates from $p_{s}(y)$ mostly at the larger sizes. The larger sizes in these distributions correspond to the larger particles of $p(R)$ appearing close to the near-end limit of the DOF in the viewable distance. As the viewable area is relatively low at the lower horizontal distance, the borescope may fail to capture some of the larger particles of the $p(R)$ which settled at the lower horizontal distance. Hence, the $p d f$ of the larger sizes in $p_{e}(y)$ is lower than that in $p_{s}(y)$. Nonetheless, $p_{s}(y)$ can be considered to be equivalent to $p_{e}(y)$. Therefore, $p_{r}(R)$ reconstructed from the respective $p_{s}(y)$ can be used to examine the validity of the proposed method at different conditions.

Effect of Depth-of-Field (DOF) distance. Generally, it is possible to change the DOF of an imaging device by changing the aperture size and/or the focusing plane location. Through an adjustment of both aperture size and focusing plane, the near-end limit and rear-end limit of the DOF can be controlled. In this section, the effect of DOF on the stability of the proposed method is examined. In this analysis, the focusing plane location is kept constant and the DOF is assumed to be changed by changing the aperture size. The relationship between magnification and distance from the borescope obtained in the calibration experiment is used for this analysis.

Three DOFs are used in this analysis, namely, 20,12 and $6 \mathrm{~mm}$. The focusing plane location is fixed at $5 \mathrm{~mm}$ from the imaging device. Since the rear DOF is normally larger than the front DOF, and the ratio of rear DOF to the front DOF increases with an increase in DOF, ${ }^{33-35}$ a rear DOF to front DOF ratio of 4, 3, and 2 are used for a DOF of 20, 12 and $6 \mathrm{~mm}$ respectively. Therefore, the viewable distance is limited within the distance of 1-21, 2-14, and 3-9 $\mathrm{mm}$ for the DOF of 20, 12 and $6 \mathrm{~mm}$ respectively.

$p_{s}(y)$ for the three different DOFs are represented by bins in Figure 9. With an increase in DOF, the average of $p_{s}(y)$ decreases. This is because if DOF is increased by keeping the focusing plane location constant, the ratio of rear DOF to front DOF also increases. As objects in the rear DOF have lower magnification, $p_{s}(y)$ is shifted towards the lower size range with the increase in DOF and hence, has lower average size. As both of the front and 
rear DOF increase with the increase in DOF, objects are subjected to a larger range of magnifications. Therefore, the variance of $p_{s}(y)$ also increases with the increase in DOF. The cumulative square error of the $p_{s}(y)$ also increases with the increases in DOF as shown in the Table 2. However, for a very shallow DOF, since the overall difference in magnification in the viewable distance is relatively small, the cumulative square error would be within a tolerable limit. Hence, the $p_{e}(y)$ would still be a reasonable representation of the $p(R)$. Nonetheless, it is possible to obtain $p(R)$ for all the DOFs after reconstruction with the proposed method. It is also found that in this theoretical analysis the cumulative square error of the $p_{r}(R)$ is low compared to that of experimental. This is due to the fact that the theoretical analysis follows all the assumptions made in this proposed method whereas experiment may have slight deviation from the assumed conditions. However, the cumulative square error of $p_{r}(R)$ under experimental condition is still low enough for a good estimation of $p(R)$.

Effect of DOF Accuracy. DOF is an important parameter needed in the determination of $p_{r}(R)$. As DOF is an analog parameter, the determination of the actual DOF may not be possible. Therefore, the effect of DOF accuracy on $p_{r}(R)$ is analyzed theoretically. In this analysis, a DOF of $20 \mathrm{~mm}$ is used for the generation of $p_{s}(y)$. However, the reconstruction of $p_{r}(R)$ is performed using three different DOFs. One DOF overestimates, one DOF underestimates and one uses the actual DOF.

$p_{r}(R)$ reconstructed with DOFs of $16.25,20$, and $23.75 \mathrm{~mm}$ and the corresponding $p_{s}(y)$ and $p(R)$ are shown in Figure 11. It can be seen that the variance of $p_{r}(R)$ does not seem to be a strong function of DOFs used in the in the reconstruction. However, an underestimation of the DOF $(16.25 \mathrm{~mm})$ would yield a $p_{r}(R)$ that has a smaller average than $p(R)$ while an overestimation of the DOF $(23.75 \mathrm{~mm})$ would yield a $p_{r}(R)$ that has a larger average than $p(R)$. This is because the average size is dominating by the magnification at the rear DOF where the number of objects present in the region is larger. Nonetheless, $p_{r}(R)$ is able to give a better representation of $p(R)$ compared to $p_{s}(y)$. As shown in Table 3, a substantial reduction in the cumulative error can still be obtained. However, the analysis result indicates that an accurate determination of the DOF limits is particularly important.

Effect of Degree of Change in Magnification. For a particular focusing plane location, the degree of change in magnification with distance in the viewable distance can vary depending on the design and number of lens in the imaging device, lens focal length as well as the refractive index of lens material and the surrounding fluid medium etc. Hence, the effect of the degree of change in magnification on the stability of the proposed method is examined. However, the location of the focusing plane can also vary depending on the application. Normally in in-situ visualization (borescope, particle video microscopy etc), objects are close to the lens and hence, the focusing plane location is near the imaging device. On the other hand, in images captured by normal lens, the objects and hence, the focusing plane is normally far away from the lens. Therefore, the stability of the proposed method with different focus adjustments is also examined.

Five different magnification curves are shown in Figure 11. Line-1 shows no change in magnification with distance which corresponds to the special case of telecentric lens. Measurements taken with telecentric lens would give the actual size distribution directly. However telecentric lenses tend to be large and more expensive than the normal lenses. Hence, the application of telecentric lens is still limited mainly due to those physical constraints. Two additional lines are generated by taking different values of $k_{1}$ and $k_{2}$ in eq 4 to evaluate the effect of the degree of change in magnification on the accuracy of the 
proposed method. Line-2 has relatively lower degree of change in magnification whereas line-3 has relatively higher degree of change in magnification. The focusing plane location is at $5 \mathrm{~mm}$ for both conditions. The DOF for both conditions are also assumed to be the same at 1-21 mm. $p_{s}(y)$ and $p_{r}(R)$ for the different degrees of change in magnification are compared in Figure 12. As shown in Figure 11, a high degree of change in magnification would cause the magnification at the front DOF to increase while the magnification at the rear DOF will decrease. This would generate object images with wider size range and thus increases the variance of $p_{s}(y)$. Since larger amount of objects will be affected by the reduction in magnification at the rear DOF, the average of $p_{s}(y)$ also decreases when the degree of change in magnification is increased. Therefore, the cumulative square error increases with the increases in the degree of change in magnification as shown in Table 4. As a result, $p_{e}(y)$ becomes more unreliable when the degree of change in magnification is high. It can also be seen from Table 4 that the cumulative square error is zero for $p_{r}(R)$ of these two degrees of change in magnification. Therefore, the proposed method is valid at any degree of change in magnification with distance.

Though the focusing plane is usually close to the imaging device in in-situ visualization, other imaging device may make use of a focusing plane far from the imaging device. Therefore, the stability of the proposed method is also investigated with a different focusing plane location. In addition to the focusing plane located at $5 \mathrm{~mm}$, a focusing plane located at $15 \mathrm{~mm}$ is also used with the same $\left(k_{1}\right.$ and $\left.k_{2}\right)$ for line-2 and line-3. It is to note that line-4 has the same $k_{1}$ and $k_{2}$ as line- 2 while line-5 has the same $k_{1}$ and $k_{2}$ as line-3. In this analysis, the viewable distance is kept limited at $1-11 \mathrm{~mm}$ when the focusing plane is at $5 \mathrm{~mm}$. The viewable distance is from 11-21 mm when the focusing plane is located at $15 \mathrm{~mm}$. The DOF and the rear-DOF to front-DOF ratio (a value of 1.5 in this case) are kept constant for all the conditions. As shown in Figure 11, as the distance between the imaging device and the focusing plane increases, the degree of change in magnification decreases.

$p_{s}(y)$ is compared with the respective $p_{r}(R)$ and $p(R)$ for both conditions of different degree of change in magnification in Figure 13 and 14. Since a change in focusing plane location from $5 \mathrm{~mm}$ to $15 \mathrm{~mm}$ essentially reduces the degree of change in magnification, the result is practically the same as decreases the degree of change in magnification by using a lens with different magnification factor. As shown in the Table 5, the cumulative square error for 15 $\mathrm{mm}$ is significantly lower than the cumulative square error for $5 \mathrm{~mm}$ for both conditions of magnification. Hence, it is possible to increase the reliability of $p_{e}(y)$, to some extent, by using a farther focusing plane location. However, if the object concentration is high, visualization may not be possible in the targeted region. For both focus adjustment, $p_{r}(R)$ has zero cumulative square error and completely resemble to $p(R)$. Therefore, focus adjustments or the degree of change in magnification has no effect on the accuracy of the proposed method.

Effect of Number of Bins. The proposed method takes into account that there are $M$ evenly spaced bins with midpoint sizes, $R_{j=1, \ldots, M}$ in $p(R)$. It is also corresponding to $M$ number of bins for the reconstructed distribution. It is important to ensure that the number of bins is sufficient to describe $p(R)$. Hence, the effect of the number of bins on the accuracy of $p_{r}(R)$ is also examined.

The effect of the number of bins is evaluated through the reconstruction of actual object size distribution from a given $p_{s}(y)$ using different numbers of bins. The same size range as $p_{s}(y)$ of $0-7 \mathrm{~mm}$ is used for the reconstruction of $p(R)$. The reconstruction is performed for three 
different bin numbers, namely, 7 ( 1 bin per $\mathrm{mm}), 14$ ( 2 bins per $\mathrm{mm})$, and 56 ( 8 bins per $\mathrm{mm}) . p_{r}(R)$ for these three bin numbers are compared in Figure 15. It can be seen from Table 6 that $p_{r}(R)$ with 56 bins has the highest accuracy though the accuracy is also acceptable for the 7 and 14 bins. For a normal distribution, Sturges proposed the following equation: ${ }^{43}$

$$
M=1+\ln (x)
$$

where $M$ is the number of bins and $x$ is the number of object sizes obtained from images. Since the lowest number of bins used in this study is 7 and the reconstructed object size distribution $p_{r}(R)$ can match the actual object size distribution $p(R)$ with reasonable accuracy, it is recommended that the minimum number of bins needed in the reconstruction would be the maximum value between 7 and $M$ determined by eq 12. More robust methods to determine the number of bins may also be used if better representation of the distribution is needed.

Selection of Distribution Function. In this proposed method, a continuous distribution function needs to be assumed for $p(R)$. Selection of an appropriate distribution function depends on the system and specific applications. Prior knowledge of a system would help to identify a suitable distribution function. For example, a log-normal distribution function is commonly used to fit the bubble size distribution in a bubble column reactor. However, the selected distribution function may not be the best distribution function to represent $p(R)$ in a system. Hence, the accuracy of the proposed method is also tested theoretically when the selected distribution function is not the same as the actual distribution function.

In this analysis, $p(R)$ is assumed to follow a two-parameter gamma distribution function:

$$
p(R \mid s, t)=R^{s-1} \frac{e^{-\frac{R}{t}}}{t^{s} \Gamma(s)}
$$

where $s$ is the shape parameter and $t$ is the scale parameter. As shown in Figure 16, depending on the values of the parameters, gamma distribution function can exhibit three different characteristics, namely symmetric, asymmetric and monotonic. Therefore, all the three characteristics gamma distribution functions are assessed in this section. $p_{s}(y)$ is simulated for these three gamma distributions in the same way described before. A lognormal distribution function is used to reconstruct each of the known $p(R)$ from the corresponding $p_{s}(y)$.

For symmetrical gamma distribution, $p_{s}(y), p_{r}(R)$, and $p(R)$ are compared in Figure 17. It can be seen that $p_{r}(R)$ reconstructed using the log-normal distribution function can closely resemble $p(R)$ which is a symmetrical gamma distribution. As indicated in Table 7, a substantial reduction in cumulative square error is achieved in the reconstruction process.

Asymmetrical distribution is more common than the symmetrical distribution in practice. Hence, a highly skewed asymmetrical gamma distribution is tested. As can be seen from Figure 18 that though $p_{r}(R)$ reconstructed using log-normal distribution function deviates from the actual gamma distribution to some extent, it is still within a reasonable limit.

Another type of gamma distribution where the probability density function decreases monotonically with size is also analyzed. As shown in Figure 19, the assumption of lognormal distribution function for $p(R)$ does not provide a good agreement between $p_{r}(R)$ and $p(R)$. Table 7 also shows that the log-normal assumption of a monotonic gamma distribution function would yield a cumulative square error two orders of magnitude higher than that of asymmetric or symmetric gamma distribution. Therefore, the assumption of a correct 
distribution function is important to the accuracy of $p_{r}(R)$. In fact, $p_{e}(y)$ would be able to give a reasonable approximation on the type of distribution for $p(R)$. For the case of monotonic gamma distribution, it can be seen in Figure 19 that $p_{s}(y)$ is heavily skewed. A distribution that has this character should then be adopted in the reconstruction.

\section{Concluding Remarks}

Most size measurements from image analysis make use of a single calibration scale. However, if the objects are not all on the same plane, the use of a single calibration scale would post an error in the size measurement. A statistical method is employed to convert the size distribution obtained from image analysis to the actual object size distribution. The method has successful been tested on the particle size distribution determined in a settling system. Theoretical analysis has also been performed. The proposed method is found stable for imaging devices with different DOF, focusing location, and change in magnification with distance. However, the determination of the DOF is particularly important for an accurate reconstruction result with the proposed method.

\section{Acknowledgement}

Support by AcRF Tier1 grant RG41/06 and A*STAR SERC grant 0621010035 is gratefully acknowledged.

\section{Notation}

$\begin{array}{lll}f & = & \text { lens focal length } \\ k & = & \text { constant in location probability density function } \\ k_{1} & = & \text { magnification model parameter } \\ k_{2} & = & \text { magnification model parameter } \\ M & = & \text { number of bins } \\ M & = & \text { magnification } \\ N & = & \text { number of equally spaced sections in the viewable distance } \\ O & = & \text { center of lens } \\ p(R) & = & \text { actual object size distribution } \\ p_{r}(R) & = & \text { reconstructed object size distribution } \\ p(y) & = & \text { theoretical object size distribution obtained from images } \\ p_{e}(y)= & \text { experimental object size distribution obtained from images } \\ p_{s}(y)= & \text { object size distribution obtained from simulation } \\ p(z)= & \text { location probability density function } \\ R & = & \text { actual size of an object } \\ s & = & \text { shape parameter of gamma distribution function } \\ t & = & \text { scale parameter of gamma distribution function } \\ v & = & \text { image distance in the single lens system } \\ x & = & \text { sample size of measurement } \\ y & = & \text { object size obtained from image } \\ y_{w} & = & \text { size of well focused image in the single lens system } \\ z & = & \text { horizontal distance of an object from the imaging device. } \\ z_{F} & = & \text { focus plane location } \\ z_{n} & = & \text { near-end limit of the DOF } \\ z_{r} & = & \text { rear-end limit of the DOF }\end{array}$




\section{Greek Letters}

$\mu=$ parameter of the log-normal distribution function

$\sigma=$ parameter of the log-normal distribution function

\section{Literature Cited}

1. Tayali, N.E.; Bates, C.J. Particle sizing techniques in multiphase flows: A review. Flow Measurement and Instrumentation 1990, 1, 77.

2. Boyer, C.; Duquenne, A.-M.; Gabriel, W. Measuring techniques in gas-liquid and gasliquid-solid reactors. Chemical Engineering Science 2002, 57, 3185.

3. Kougoulos, E.; Jones, A.G.; Jennings, K.H.; Wood-Kaczmar, M.W. Use of focused beam reflectance measurement (FBRM) and process video imaging (PVI) in a modified mixed suspension mixed product removal (MSMPR) cooling crystallizer. Journal of Crystal Growth 2005, 273, 529.

4. Prasser, H.-M.; Scholz, D.; Zippe, C. Bubble size measurement using wire-mesh sensors. Flow Measurement and Instrumentation 2001, 12, 299.

5. Montante, G.; Horn, D.; Paglianti, A. Gas-liquid flow and bubble size distribution in stirred tanks. Chemical Engineering Science 2008, 63, 2107.

6. Bordel, S.; Mato, R.; Villaverde, S. Modeling of the evolution with length of bubble size distributions in bubble columns. Chemical Engineering Science 2006, 61, 3663.

7. Rojas, G.; Loewen, M. R. Fiber-optic probe measurements of void fraction and bubble size distributions beneath breaking waves. Experiments in Fluids 2007, 43, 895.

8. Laakkonen, M.; Moilanen, P.; Alopaeus, V.; Aittamaa, J. Modelling local bubble size distributions in agitated vessels. Chemical Engineering Science 2007, 62, 721.

9. Homayouni, S.-S.; Mehrnia, M.-R.; Mostoufi, N.; Rajabi, M.; Yazdani, A. Bubble size distribution in oil-based bubble columns. Chemical Engineering Technology 2008, 31, 1668.

10. Behkish, A.; Lemoine, R.; Sehabiague, L.; Oukaci, R.; Morsi, B. I. Gas holdup and bubble size behavior in a large-scale slurry bubble column reactor operating with an organic liquid under elevated pressures and temperatures. Chemical Engineering Journal 2007, 128, 69.

11. Lee, S.; Ahn, S. A.; Meyyappan, A.; Schenker, P. Depth from magnification and bluring. SPIE. 1996, 2905,235.

12. Matsuura, A.; Fan, L.-S. Distribution of bubble properties in a gas-liquid-solid fluidized bed. AIChE Journal. 1984, 30, 894.

13. Jillavenkatesa, A.; Dapkunas, S. J.; Lum, L.-S. Particle size characterization. NIST Special Publication 2001, 1, 960.

14. Yang, G.Q.; Du, B.; Fan, L.-S. Bubble formation and dynamics in gas-liquid-solid fluidization - A review. Chemical Engineering Science 2007, 62, 2.

15. James, P. M.; Syvitski (editor). Principles, methods and application of particle size analysis. Cambridge University Press 2007, ISBN-13: 9780521044615.

16. Liu, W.; Clark, N. N. Relationships between distributions of chord lengths and distributions of bubble sizes including their statistical parameters. International Journal of Multiphase Flow 1995, 21, 1073.

17. Liu, W.; Clark, N. N.; Karamavruc, A. I. General method for the transformation of chord-length data to a local bubble-size distribution. AIChE Journal 1996, 42, 2713.

18. Santana, D.; Macias-Machin, V. Local bubble-size distribution in fluidized beds. AIChE Journal. 2000, 46, 1340. 
19. Ahmed, S. A. Automatic thresholding of gray-level pictures using two-dimensional entropy. Computer Vision, Graphics, and Image Processing 1989, 47, 22.

20. Pal, N. R.; Pal, S. K. A review on image segmentation techniques. Pattern Recognition 1993, 26, 1277.

21. Lia, C.; Zhang, X.; Dong, Y.; Liu, G.; Chen, J. Oil Contamination level measurement based on microscopic digital image processing. Proceeding of SPIE 2009, 7156.

22. Roitberg, E.; Shemer, L.; Barnea, D. Application of a borescope to studies of gasliquid flow in downward inclined pipes. International Journal of Multiphase Flow 2006, 32, 499.

23. Guevorkian, K.; Valles, Jr.; James, M. In situ imaging of micro-organisms in intense magnetic fields. Review of Scientific Instruments 2005, 76, 103706.

24. Ljunggren, M.; Jönsson, L.; Jansen, J. L. C. Particle visualisation - a tool for determination of rise velocities. Water Science and Technology 2004, 50, 229.

25. Martina, K.; Stewartb, C. V. Real time tracking of borescope tip pose. Image and Vision Computing 2000, 18,795.

26. Kumar, S.; Kusakabe, K.; Raghunathan, K.; Fan, L. -S. Mechanism of heat transfer in bubbly liquid and liquid-solid systems: Single bubble injection. AIChE Journal 1992, 38, 733.

27. Kraume, M.; Gabler, A.; Schulze, K. Influence of physical properties on drop size distributions of stirred liquid-liquid dispersions. Chemical Engineering Technology 2004, 27, 330.

28. Tatiana, G.-L.; Susana, E. P. O. Drop sizes in liquid membrane dispersions. Industrial and Engineering Chemistry Research 2000, 39, 5020.

29. Daniel, A.S.; Evans, J. W. Measurements of granular flows in two-dimensional hoppers by particle image velocimetry. Part I: experimental method and results. Chemical Engineering Science 2005, 60, 1043.

30. Lovick, J.; Mouza, A. A.; Paras, S. V.; Lye, G. J.; Angeli, P. Drop size distribution in highly concentrated liquid-liquid dispersions using a light back scattering method. Journal of Chemical Technology and Biotechnology 2005, 80, 545.

31. Angeli, P.; Hewitt, G. F. Drop size distributions in horizontal oil-water dispersed flows. Chemical Engineering Science 2000, 55, 3133.

32. Haneishi, H.; Yagihashi, Y.; Miyake, Y. A new method for distortion correction of electronic endoscope images. IEEE Transactions on Medical Imaging 1995, 14, 548.

33. Larmore, L. Introduction to photographic principles. New-York: Dover Publications, Inc $1965,2^{\text {nd }}$ ed.161.

34. Ray, S. F. The geometry of image formation. In the Manual of Photography, Photographic and Digital Imaging 2000, $9^{\text {th }}$ ed., 53.

35. Ray, S. F. Applied photographic optics. Oxford: Focal Press 2002, ISBN 0-24051540-4, $3^{\text {rd }}$ ed., 217.

36. Nargesian, F.; Darabi, A. A.; Jamzad, M. Center of confusion estimation for out-offocus images based on bispectrum. Proceedings of the International Conference on Computational Intelligence and Multimedia Applications (ICCIMA 2007) 2007, 3, 501.

37. Honkanen, M.; Saarenrinne, P.; Stoor, T.; Niinimaki, J. Recognition of highly overlapping ellipse-like bubble images. Measurement Science and Technology 2005, 16,1760 .

38. Chigier, N. Optical imaging of sprays. Progress in Energy and Combustion Science 1991, 17, 211.

39. Adrian, R. J. Particle-imaging techniques for experimental fluid mechanics. Annual Review of Fluid Mechanics 1991, 23, 261. 
40. Oberdier, L. M. Instrumentation system to automate the analysis of fuel-spray images using computer vision. ASTM Special Technical Publication 1984, 123.

41. Hall, J. M. Estimating the depth of field of a complex sensor system. Proceeding of SPIE 2003. 5074, 818.

42. Koh, K.U.; Kim, J. Y.; Lee, S.Y. Determination of in-focus criteria and depth of field in image processing of spray particles. Atomization and Sprays 2001, 11, 317.

43. Sturges, H. A. The choice of a class interval. Journal of American Statistical Association 1926, 65. 
Table 1. Comparison of cumulative square error for different columns

\begin{tabular}{clc}
\hline Setup & \multicolumn{2}{c}{ Cumulative square error } \\
\cline { 2 - 3 } & $\boldsymbol{p}_{\boldsymbol{e}}(\boldsymbol{y})$ & $\boldsymbol{p}_{\boldsymbol{r}}(\boldsymbol{R})$ \\
\hline $25 \mathrm{~mm}$ ID column & 5.320 & 0.183 \\
$140 \mathrm{~mm}$ ID column & 5.800 & 0.089
\end{tabular}

Table 2. Comparison of cumulative square error for different DOF distances

\begin{tabular}{ccc}
\hline DOF $(\mathbf{m m})$ & \multicolumn{2}{c}{ Cumulative square error } \\
\cline { 2 - 3 } & $\boldsymbol{p}_{\boldsymbol{s}}(\boldsymbol{y})$ & $\boldsymbol{p}_{\boldsymbol{r}}(\boldsymbol{R})$ \\
\hline 20 & 2.449 & 0.000 \\
12 & 1.519 & 0.000 \\
6 & 0.410 & 0.000
\end{tabular}

Table 3. Comparison of cumulative square error for reconstructions using different

\begin{tabular}{ccc}
\multicolumn{3}{c}{ DOFs } \\
\hline DOF $(\mathbf{m m})$ & \multicolumn{2}{c}{ Cumulative square error } \\
\cline { 2 - 3 } & $\boldsymbol{p}_{\boldsymbol{s}}(\boldsymbol{y})$ & $\boldsymbol{p}_{\boldsymbol{r}}(\boldsymbol{R})$ \\
& $(\mathbf{D O F}=\mathbf{2 0} \mathbf{~ m m})$ & \\
\hline 16.25 & 2.449 & 0.435 \\
20 & 2.449 & 0.000 \\
23.75 & 2.449 & 0.547
\end{tabular}

Table 4. Comparison of cumulative square errors for different degree of change in magnification with distance

\begin{tabular}{clc}
\hline Magnification & \multicolumn{2}{c}{ Cumulative square error } \\
\cline { 2 - 3 } & $\boldsymbol{p}_{\boldsymbol{s}}(\boldsymbol{y})$ & $\boldsymbol{p}_{\boldsymbol{r}}(\boldsymbol{R})$ \\
\hline Higher degree & 2.811 & 0.000 \\
Lower degree & 1.086 & 0.000
\end{tabular}

Table 5. Comparison of cumulative square error for different focus adjustments

\begin{tabular}{cccc}
\hline Magnification & $\begin{array}{c}\text { Focusing } \\
\text { plane location } \\
(\mathbf{m m} \text { from } \\
\text { borescope tip) }\end{array}$ & \multicolumn{2}{c}{ Cumulative square error } \\
\cline { 3 - 4 } & 5 & 1.399 & $p_{r}(\boldsymbol{R})$ \\
\hline High degree & 5 & 0.000
\end{tabular}




\begin{tabular}{lcll}
\hline & 15 & 1.033 & 0.000 \\
Low degree & 5 & 0.089 & 0.000 \\
& 15 & 0.054 & 0.000
\end{tabular}

Table 6. Comparison of cumulative square error for different number of bins

\begin{tabular}{ccc}
\hline Number of bins & \multicolumn{2}{c}{ Cumulative square error } \\
\cline { 2 - 3 } & $\boldsymbol{p}_{\boldsymbol{s}}(\boldsymbol{y})$ & $\boldsymbol{p}_{\boldsymbol{r}}(\boldsymbol{R})$ \\
\hline 56 & 2.449 & 0.000 \\
14 & 2.449 & 0.008 \\
7 & 2.449 & 0.015
\end{tabular}

Table 7. Comparison of cumulative square error for different gamma distributions

\begin{tabular}{ccc}
\hline $\begin{array}{c}\text { Type of gamma } \\
\text { Distribution }\end{array}$ & \multicolumn{2}{c}{ Cumulative square error } \\
\cline { 2 - 3 } & $\boldsymbol{p}_{\boldsymbol{s}}(\boldsymbol{y})$ & $\begin{array}{c}\boldsymbol{p}_{\boldsymbol{r}}(\boldsymbol{R}) \\
\text { (Using log-normal) }\end{array}$ \\
\hline Symmetrical & 1.931 & 0.003 \\
Asymmetrical & 0.349 & 0.003 \\
Monotonic & 0.914 & 0.178
\end{tabular}



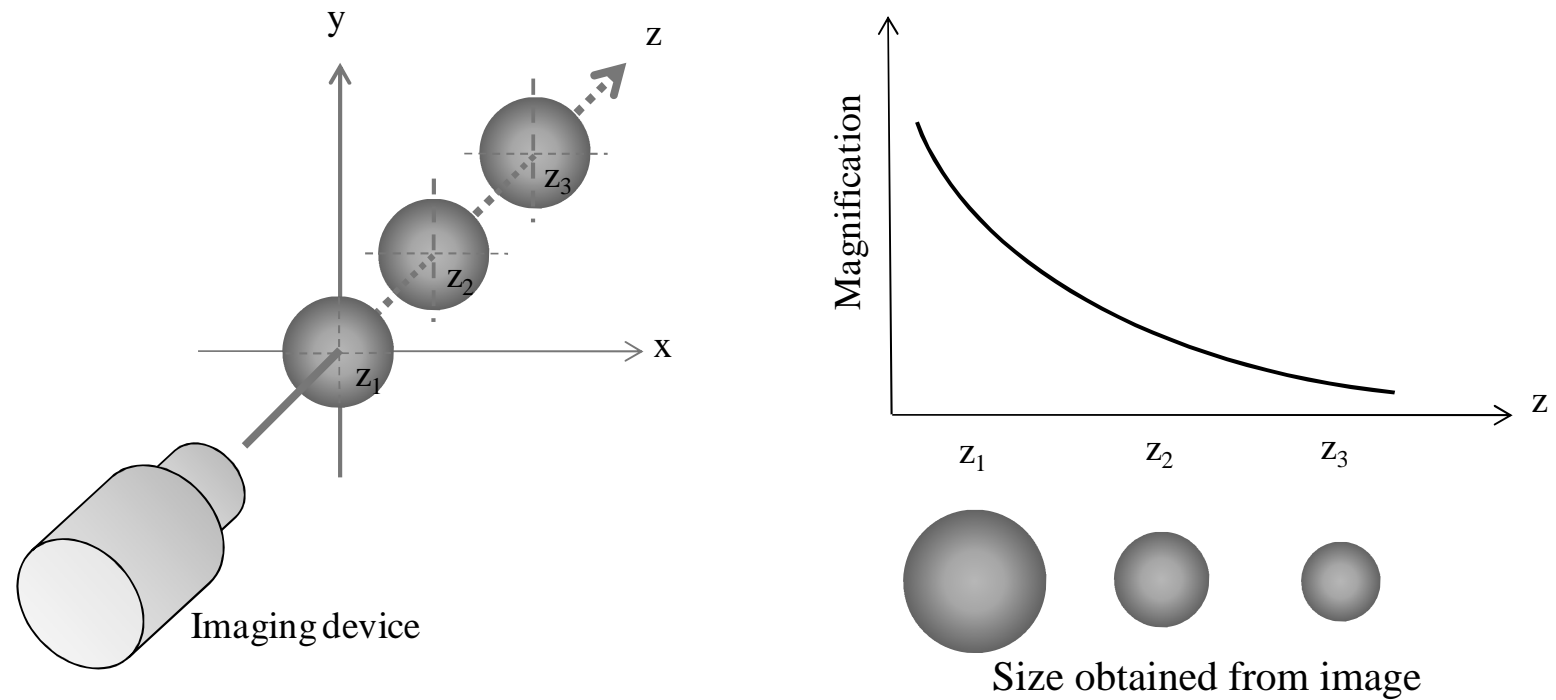

Figure 1. Effect of distance on object size obtained from image and magnification.

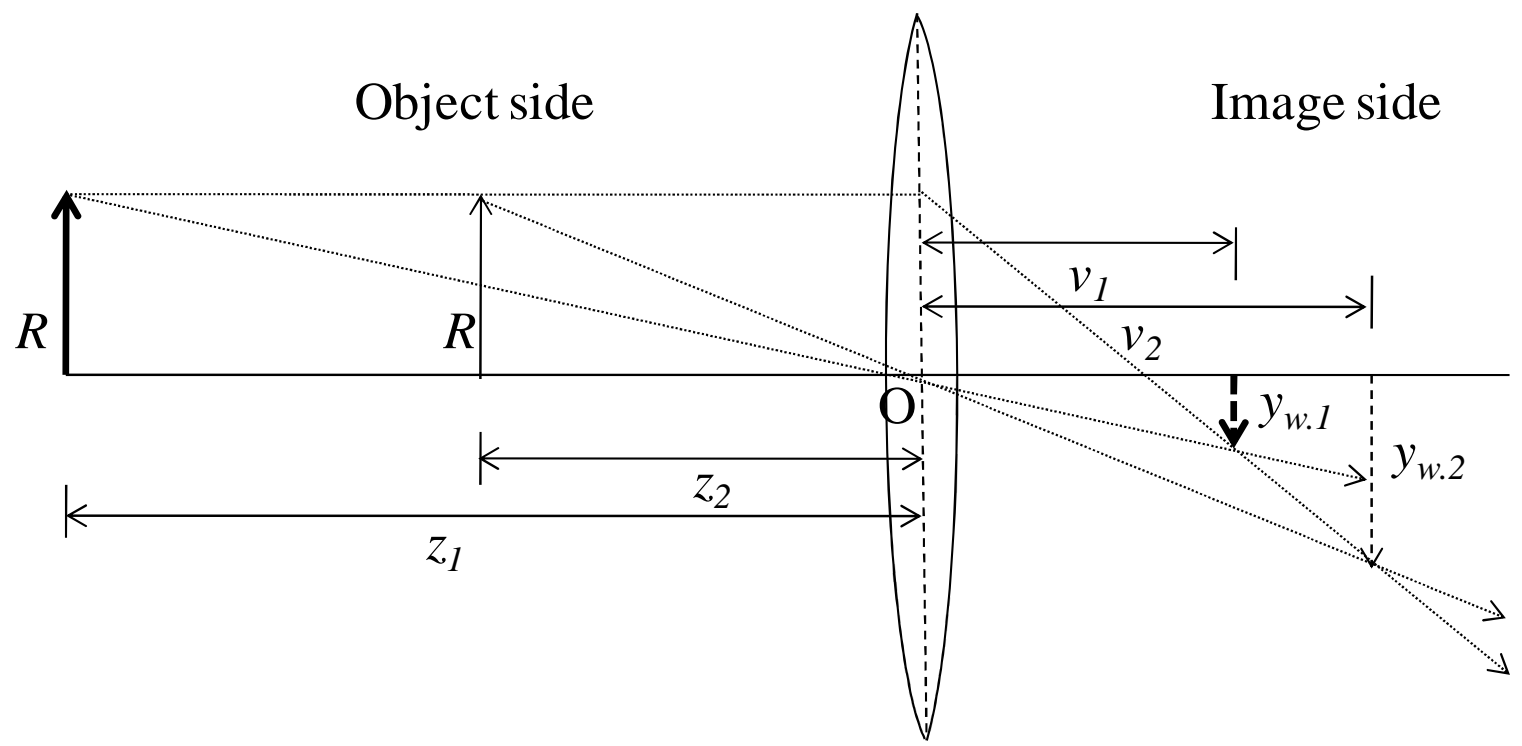

Figure 2. Image formation in a single lens system. 


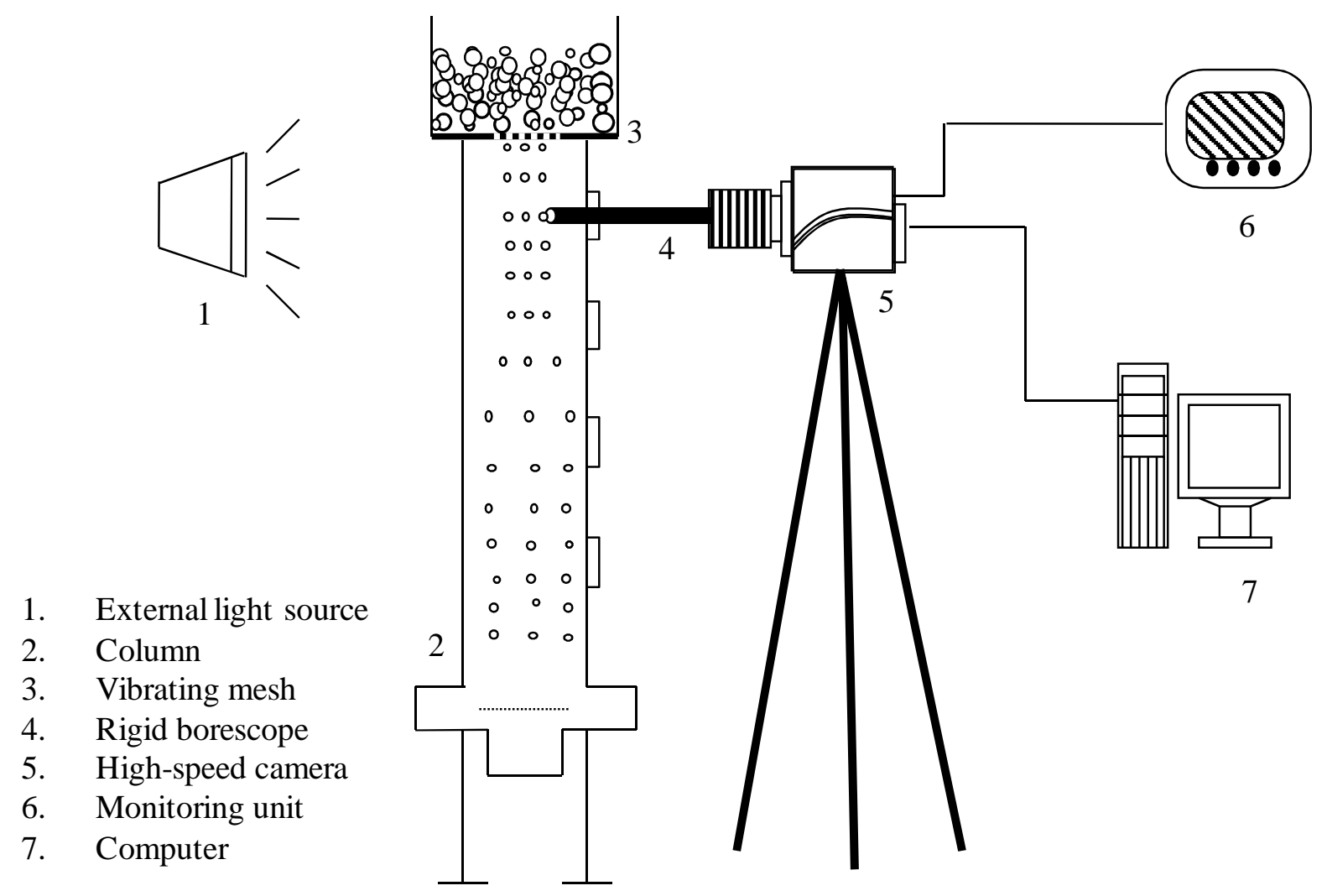

Figure 3. Schematic diagram of the experimental setup.

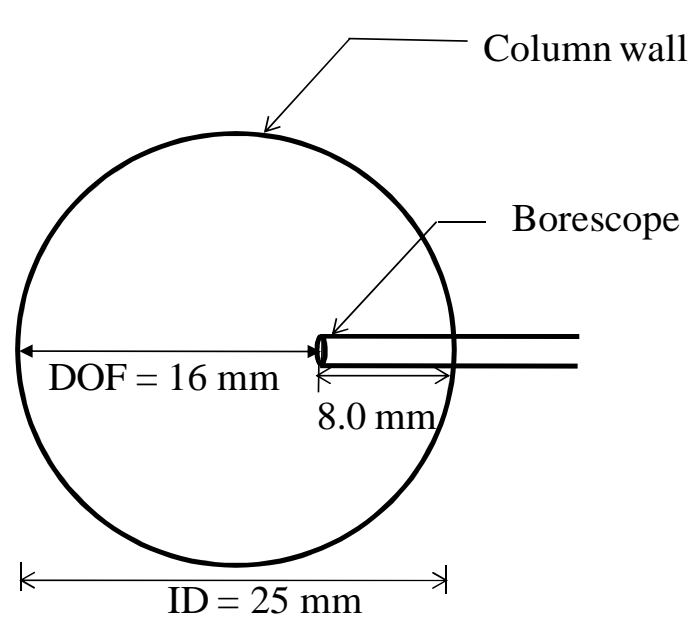

(a) DOF limited by column wall

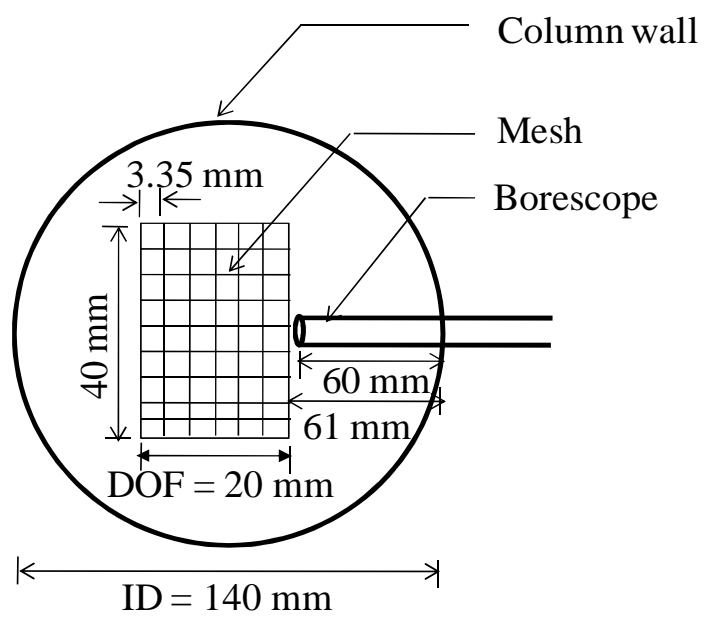

(b) DOF limited by mesh

Figure 4. Positions of borescope and mesh in the two columns. 


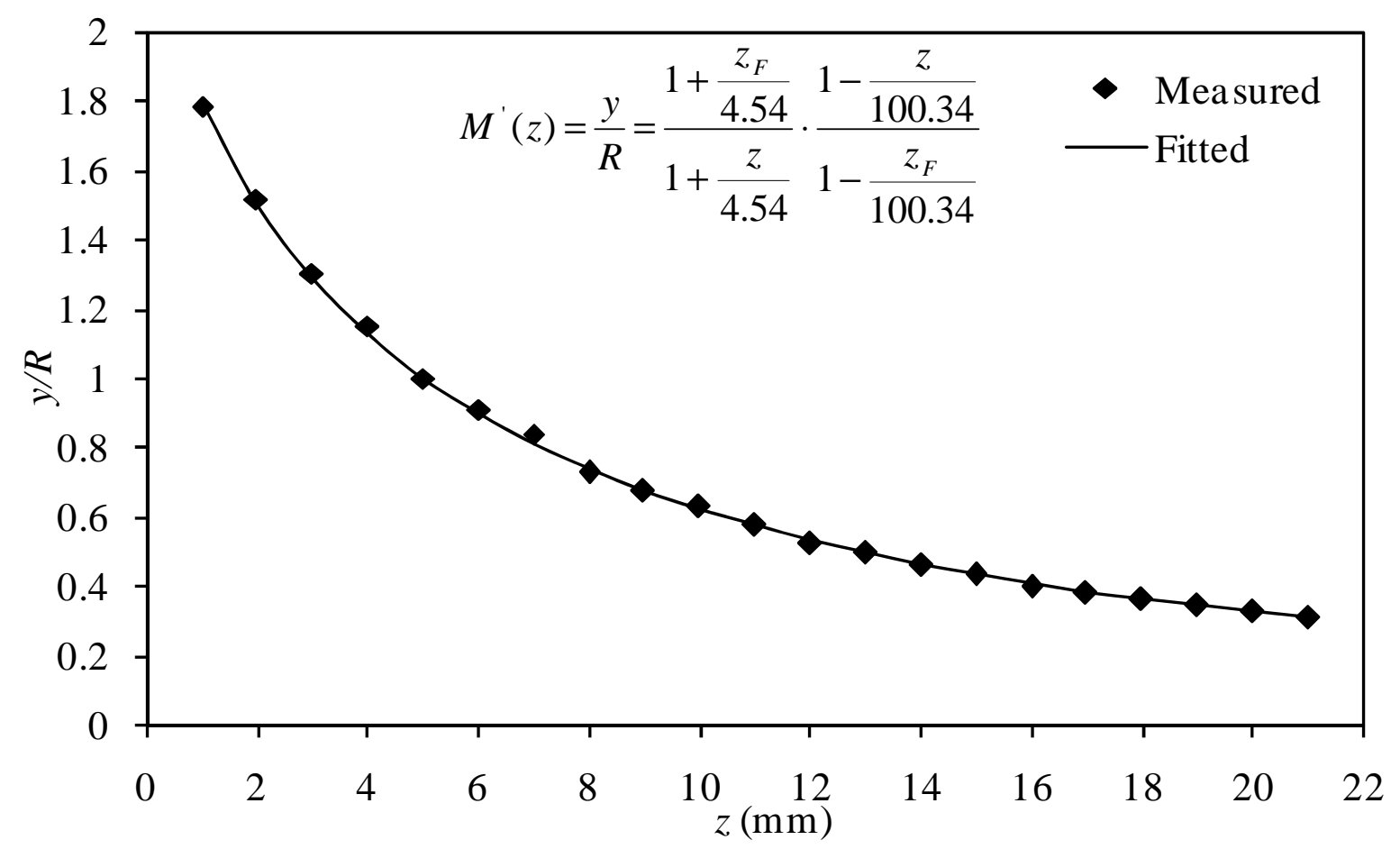

Figure 5. Magnification of borescope image at various distances from borescope tip.

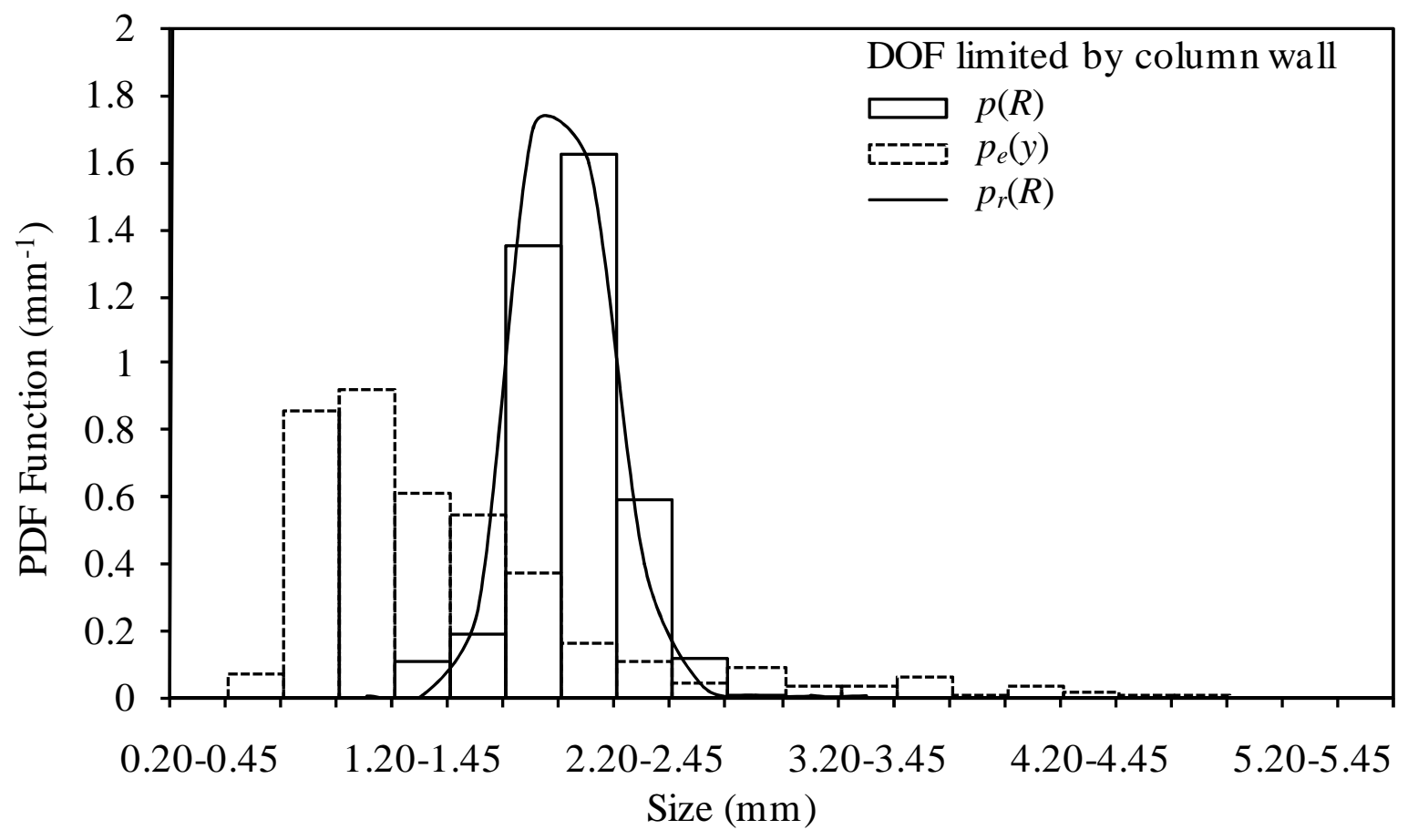

Figure 6. Comparison of the experimental particle size distribution obtained from images, the reconstructed object size distribution and actual object size distribution in a $25 \mathrm{~mm}$ ID column. 


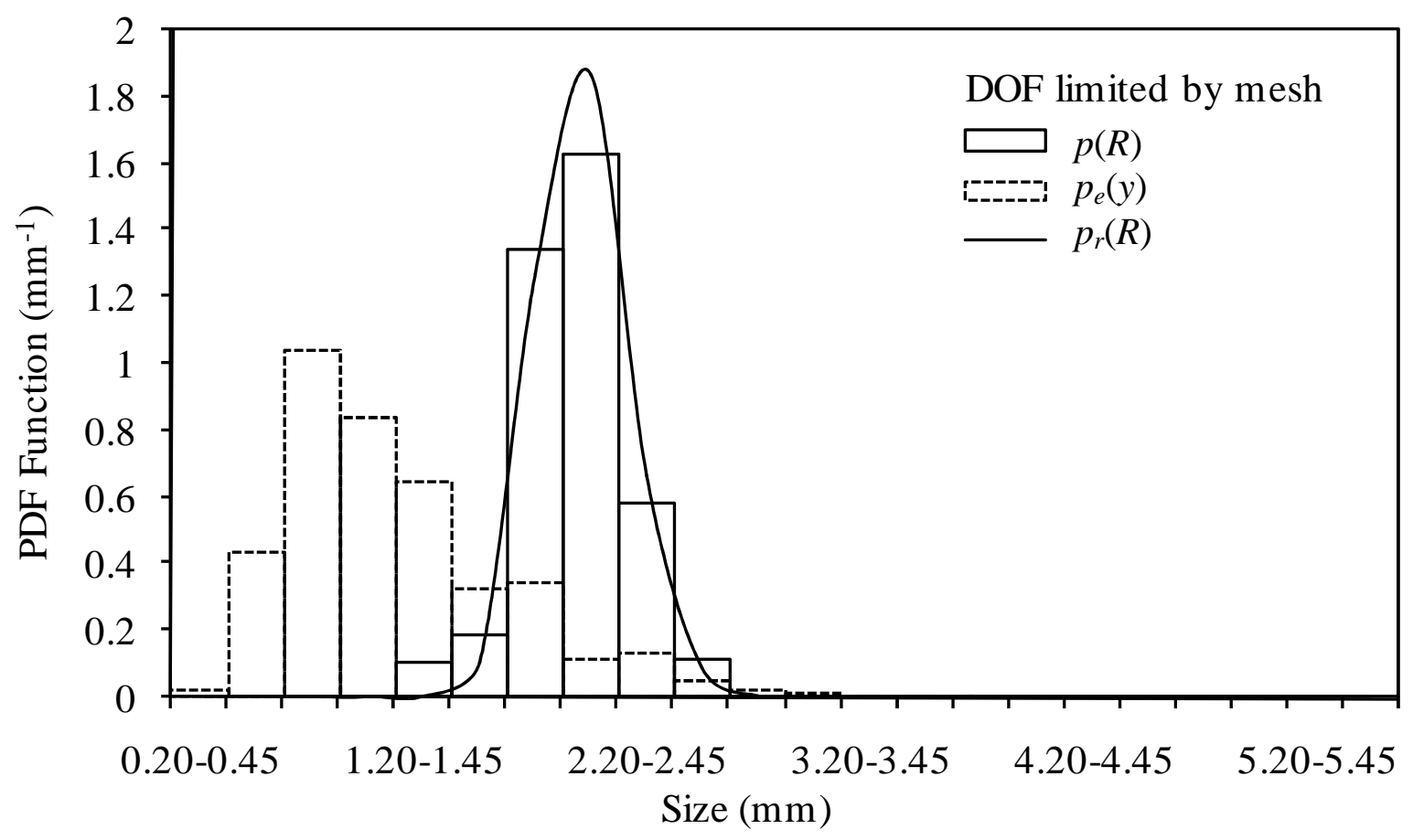

Figure 7. Comparison of the experimental particle size distribution obtained from images, the reconstructed object size distribution and actual object size distribution in a $140 \mathrm{~mm}$ ID column.

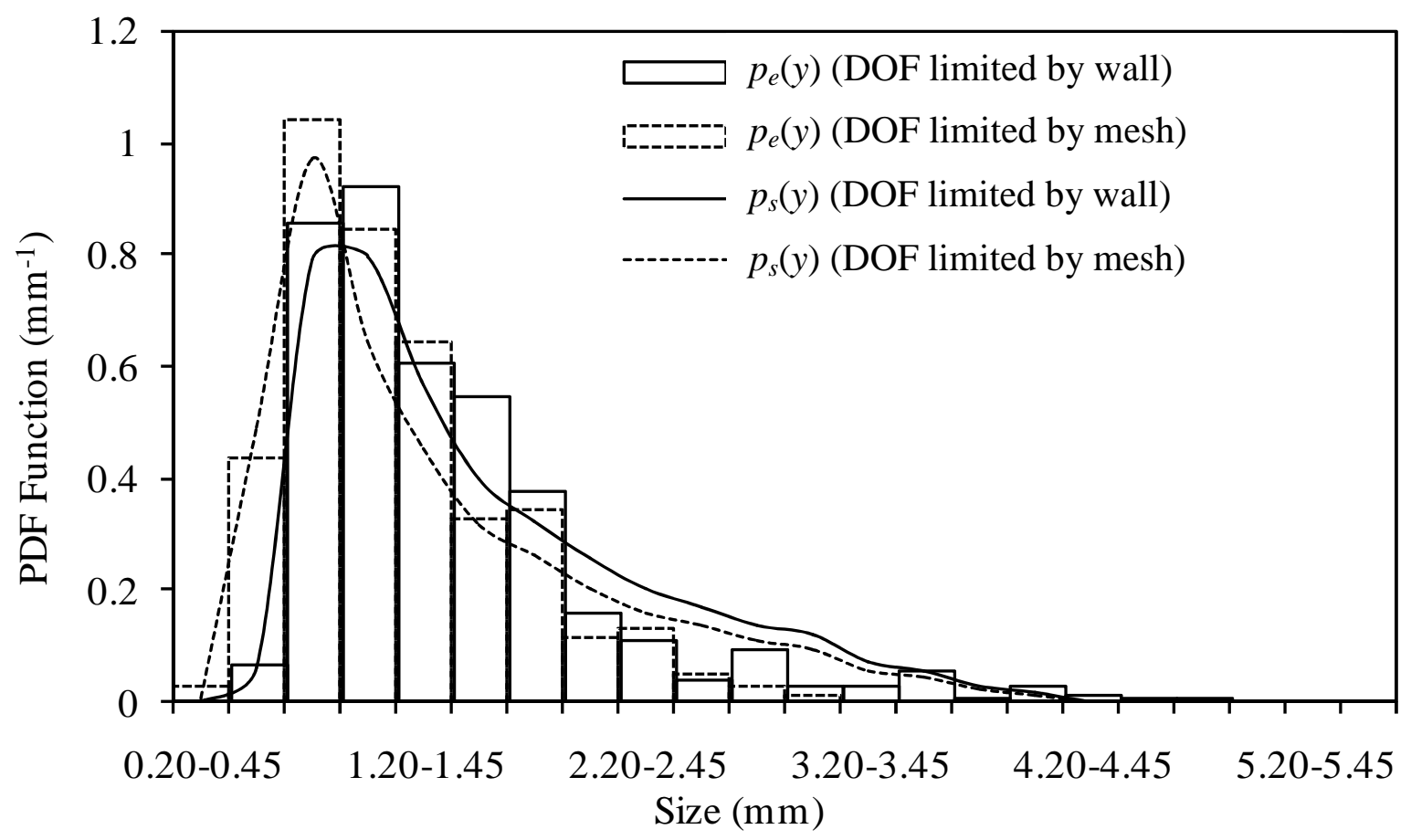

Figure 8. Comparison of the experimental object size distributions obtained from images and obtained from simulation. 


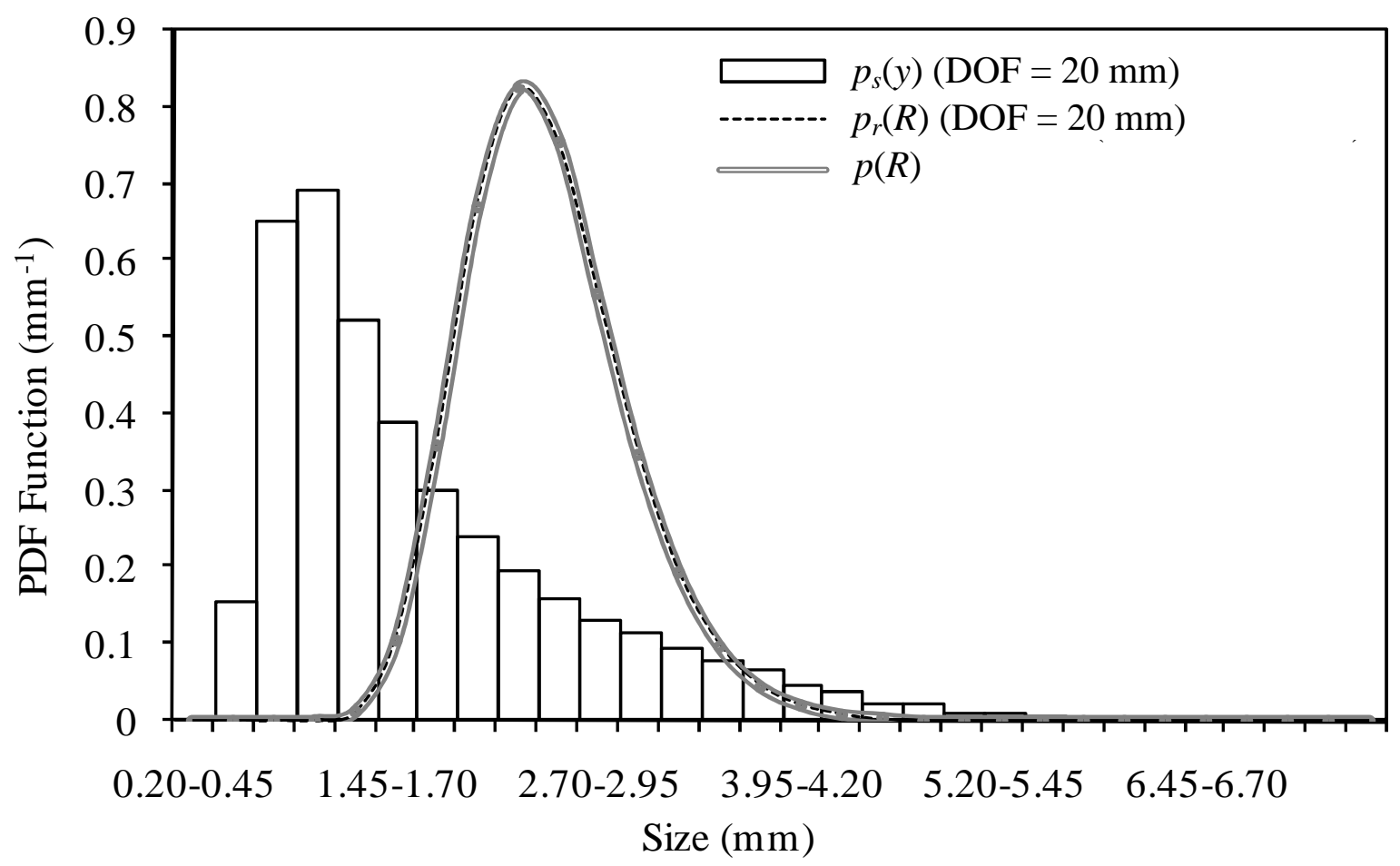

(a)

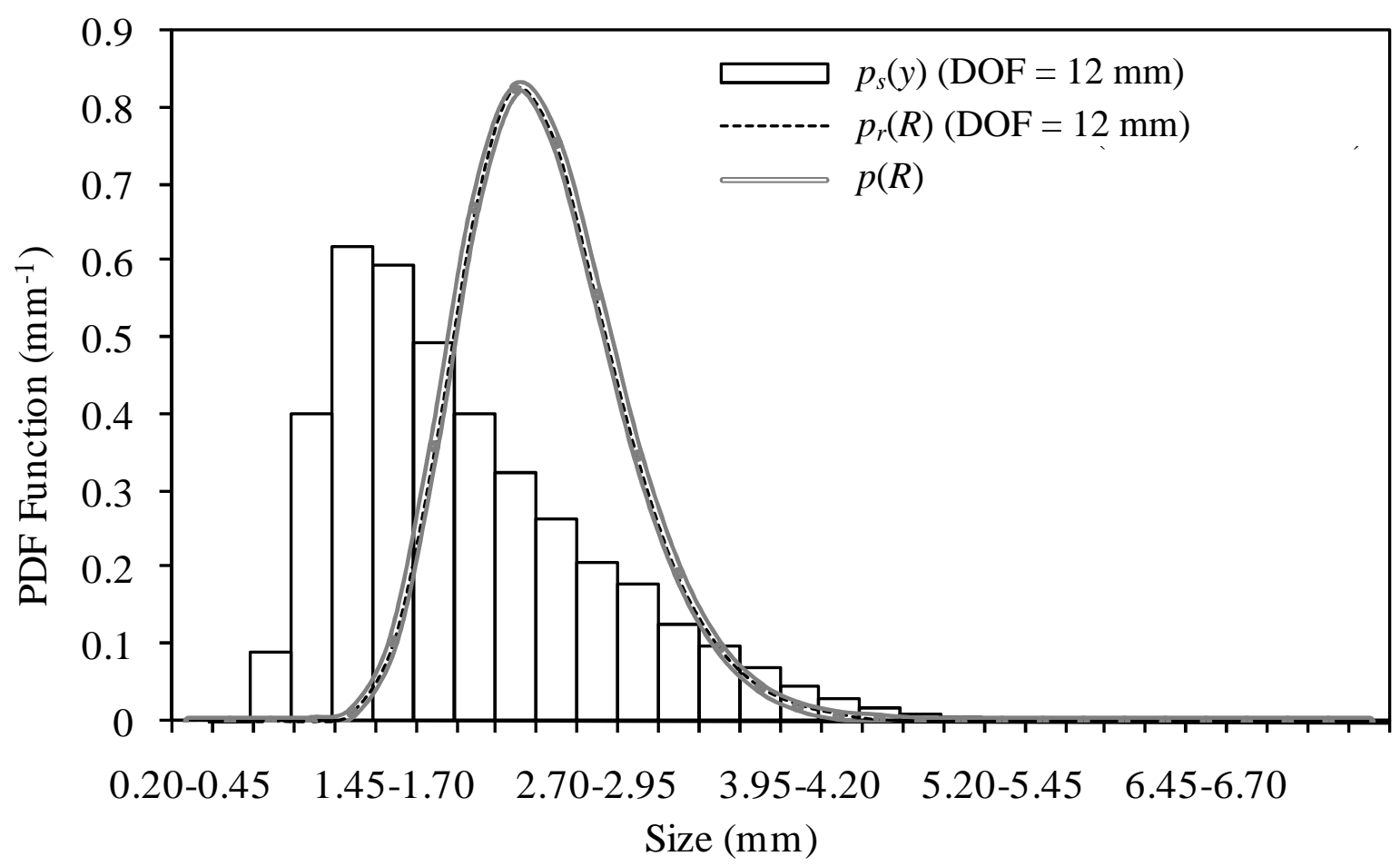

(b) 


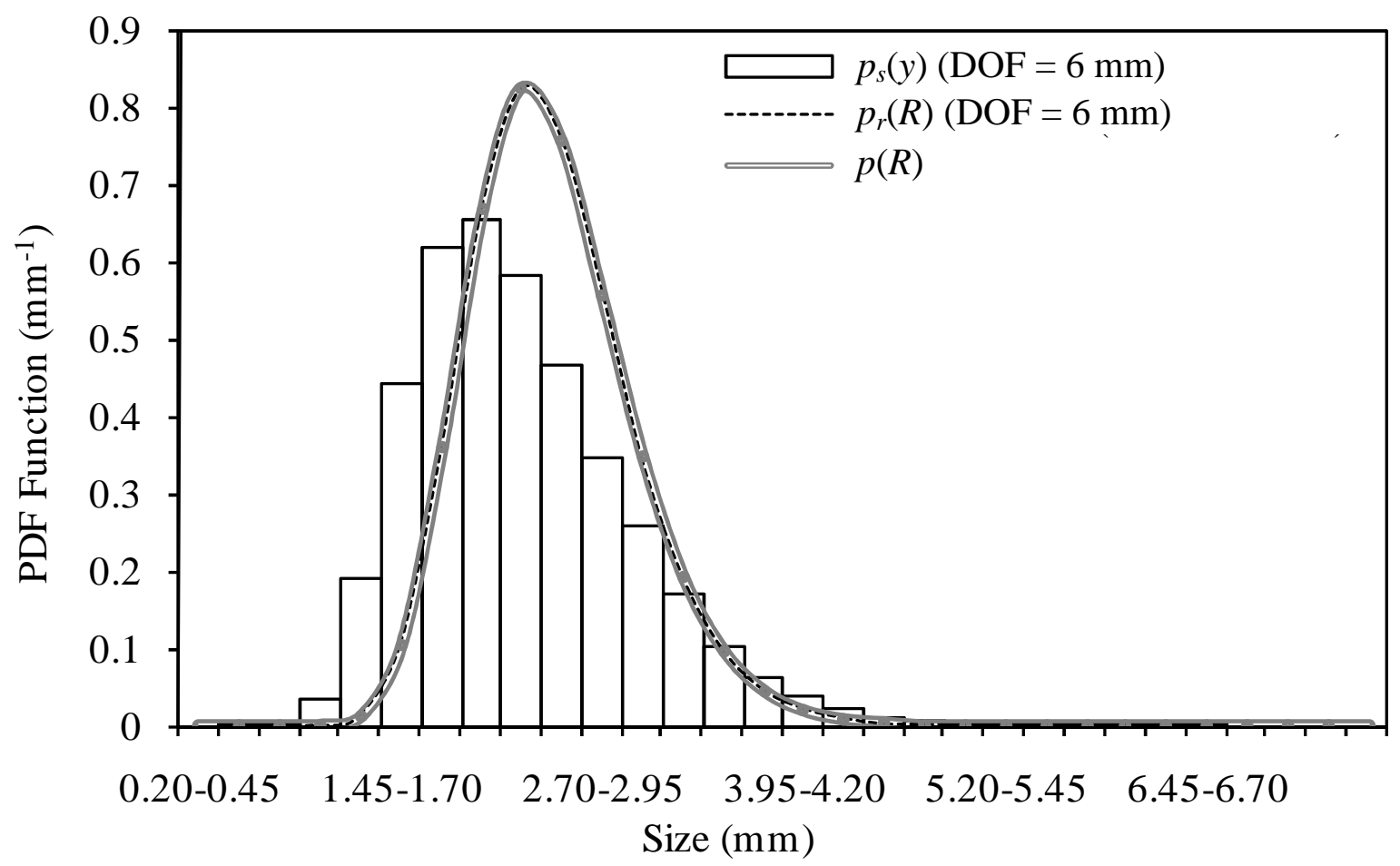

(c)

Figure 9. Evaluation of the effect of DOF distance on the stability of the proposed method, (a) DOF $=20 \mathrm{~mm}$, (b) DOF $=12 \mathrm{~mm}$, (c) DOF $=6 \mathrm{~mm}$.

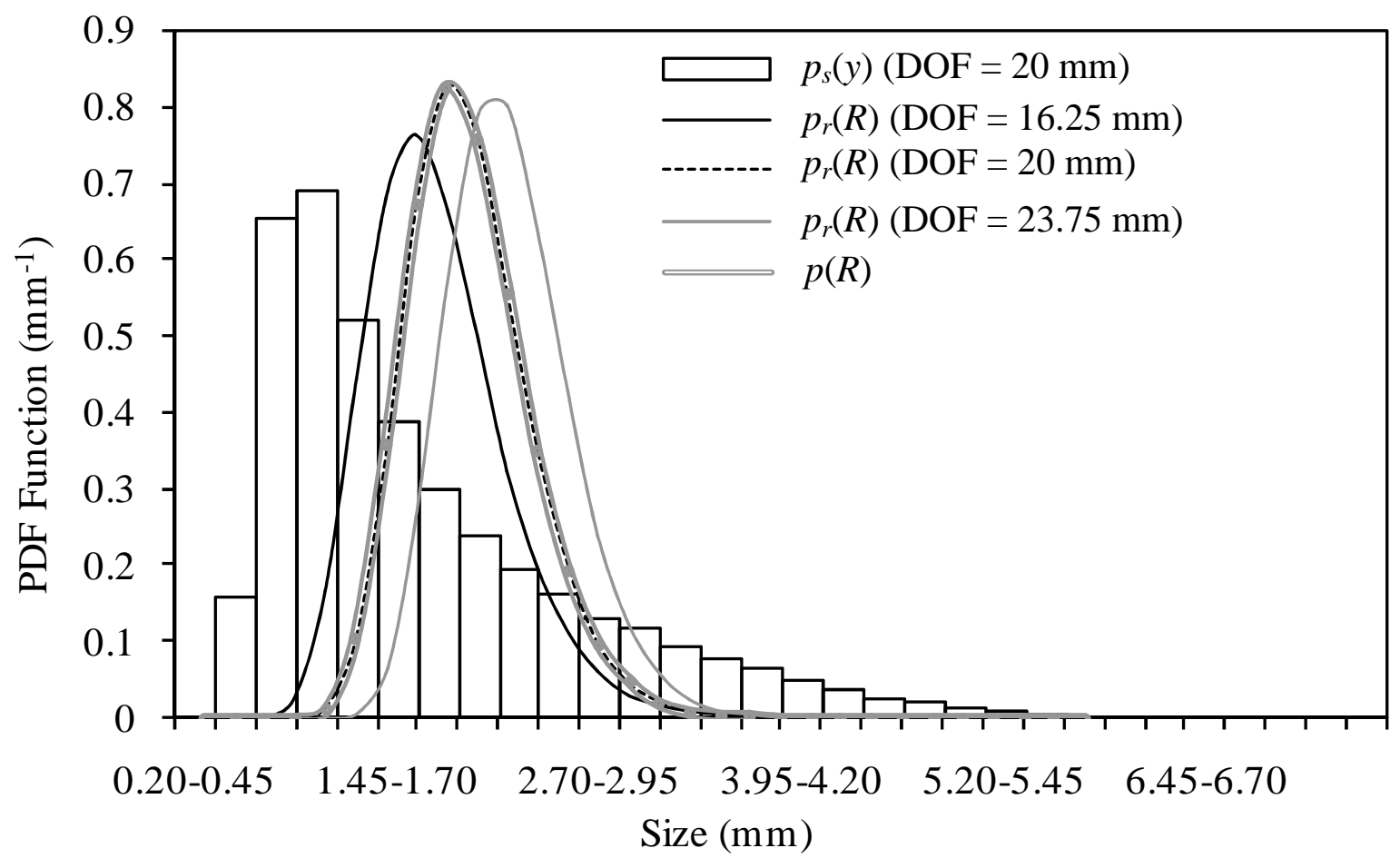

Figure 10. Evaluation of the effect of DOF used in the reconstruction. 


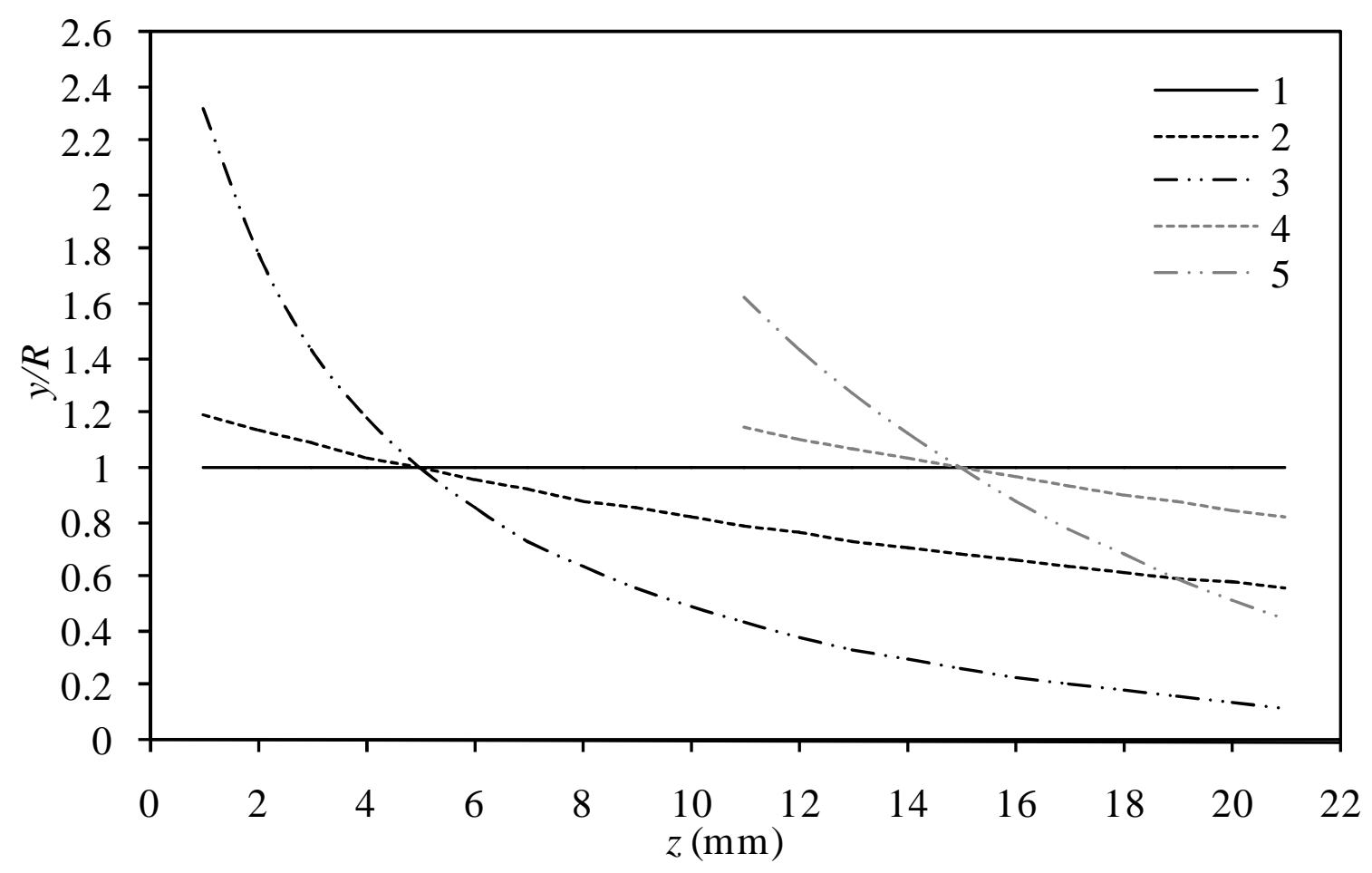

Figure 11. Different degree of change in magnification with distance. 


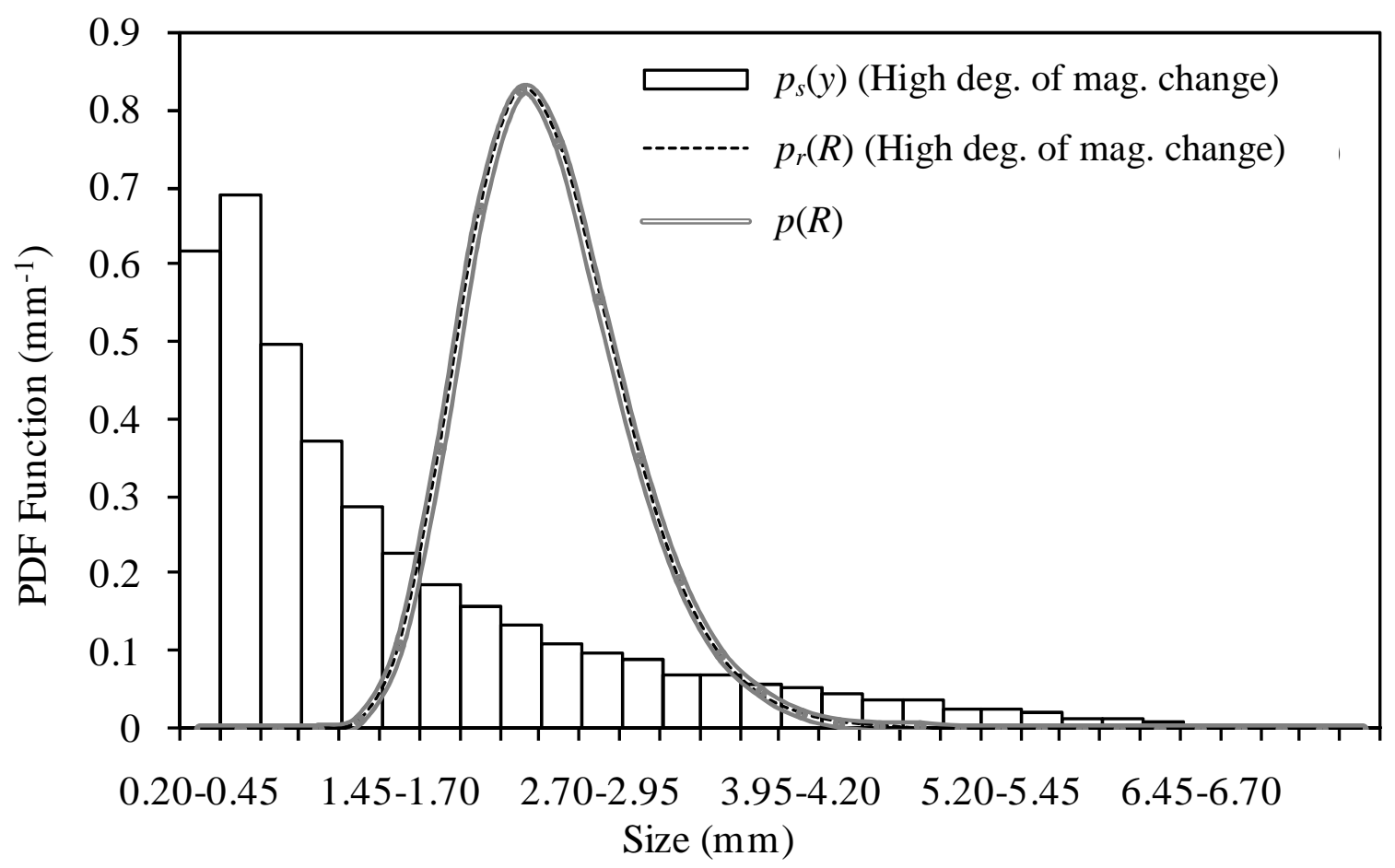

(a)

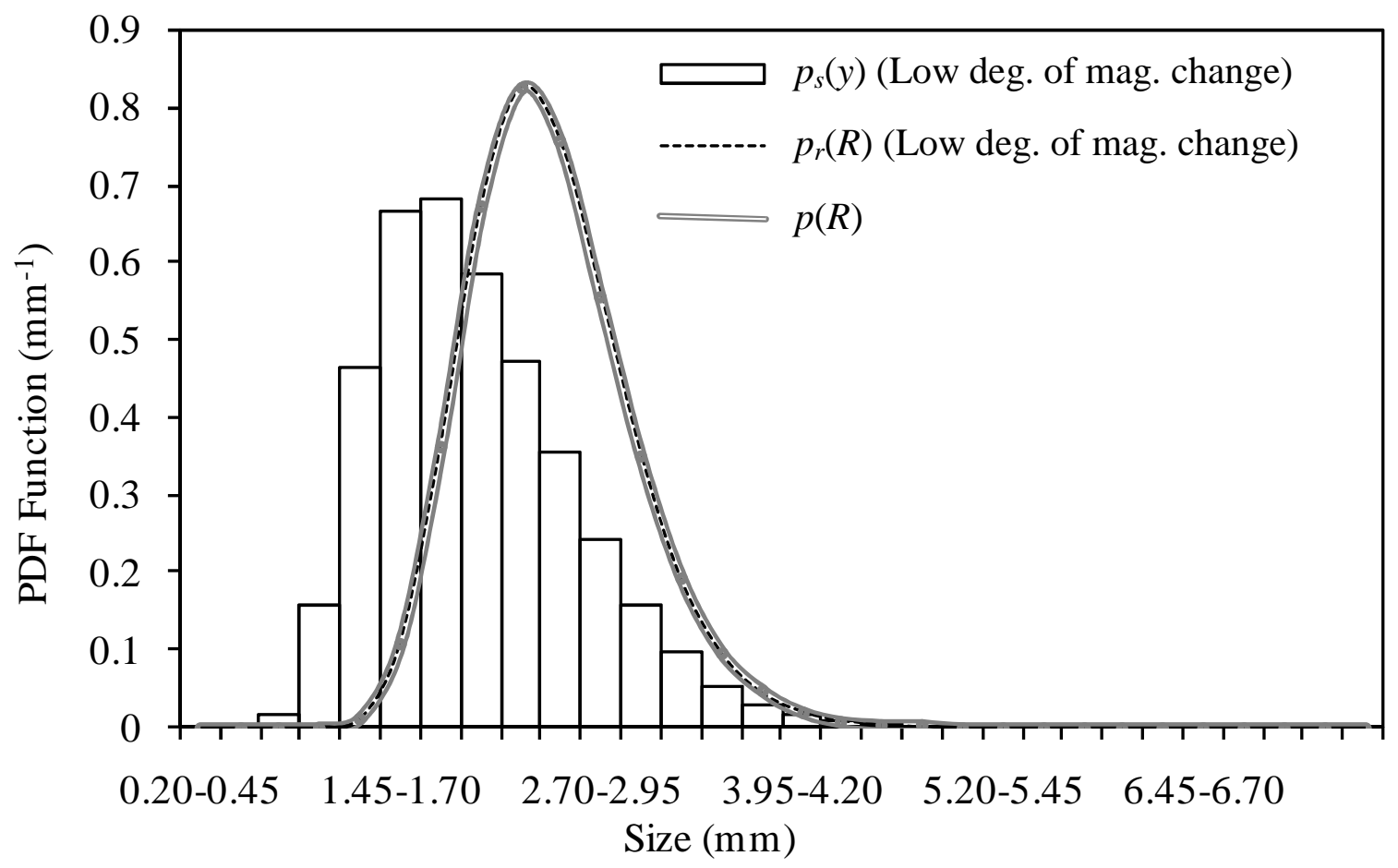

(b)

Figure 12. Evaluation of the degree of change in magnification on the stability of the proposed method, (a) high degree of change in magnification, (b) low degree of change in magnification. 


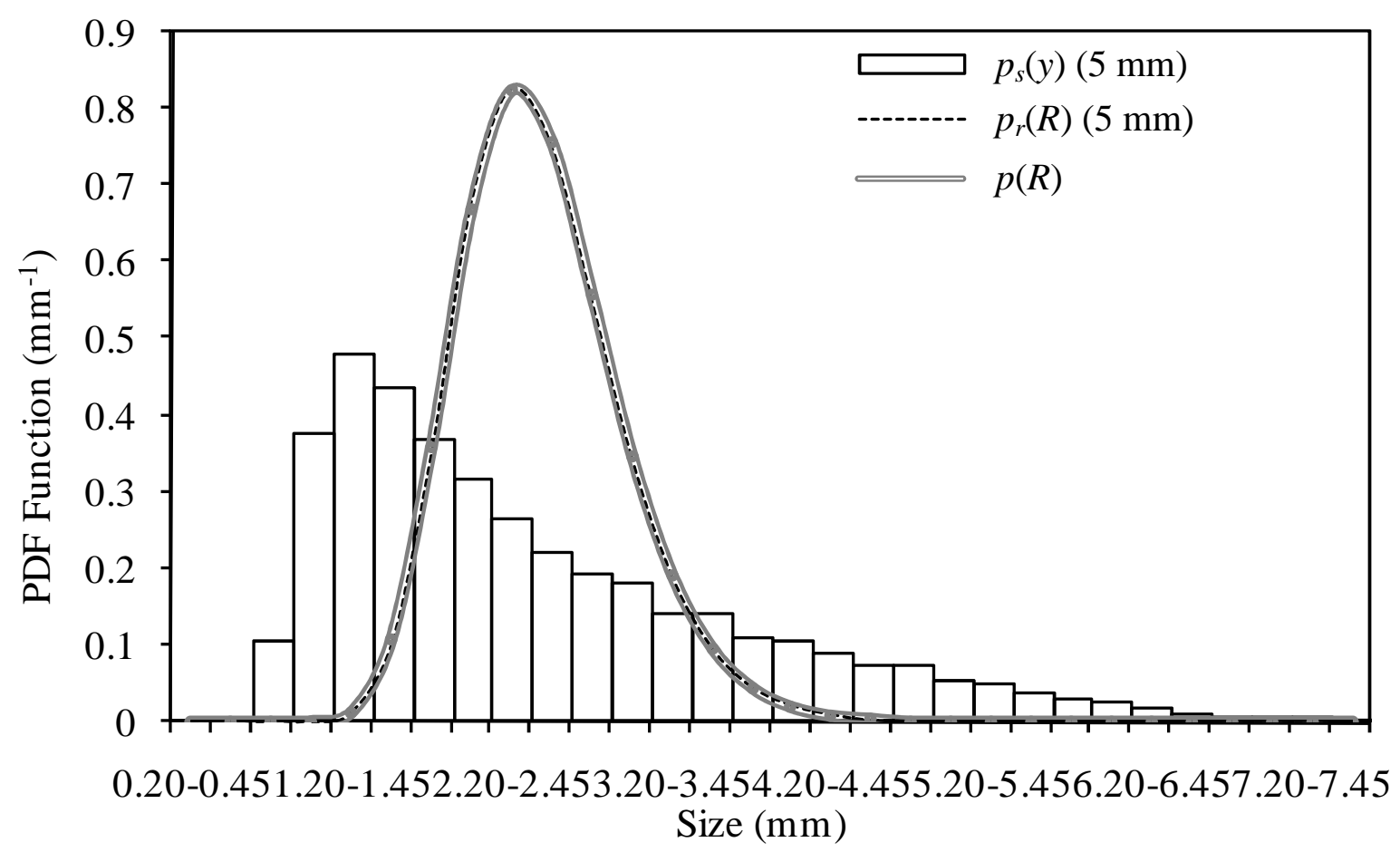

(a)

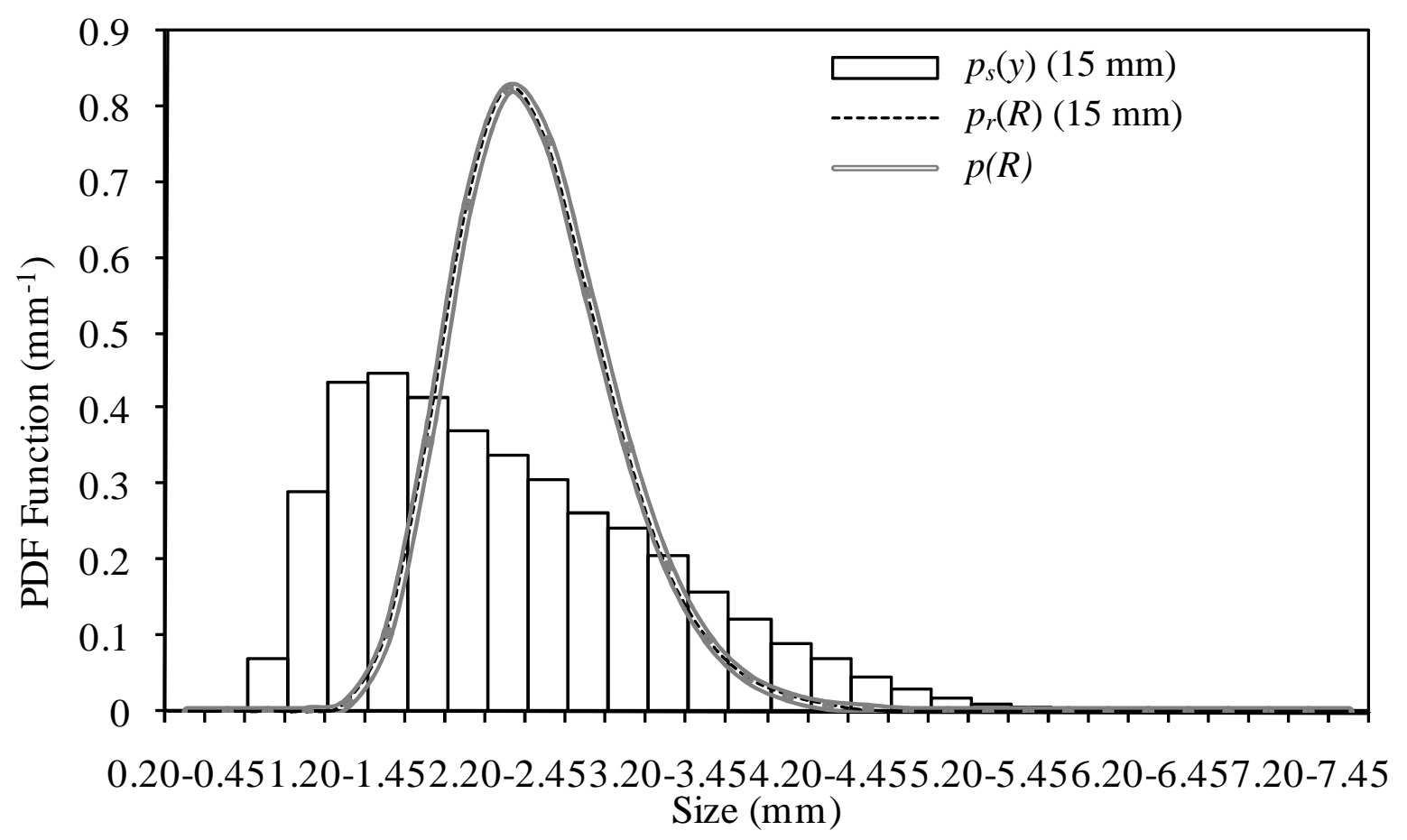

(b)

Figure 13. Evaluation of the effect of different focus adjustment on the stability of the proposed method when the degree of change in magnification is high, (a) focusing distance = $5 \mathrm{~mm}$ from borescope, (b) focusing distance $=15 \mathrm{~mm}$. 


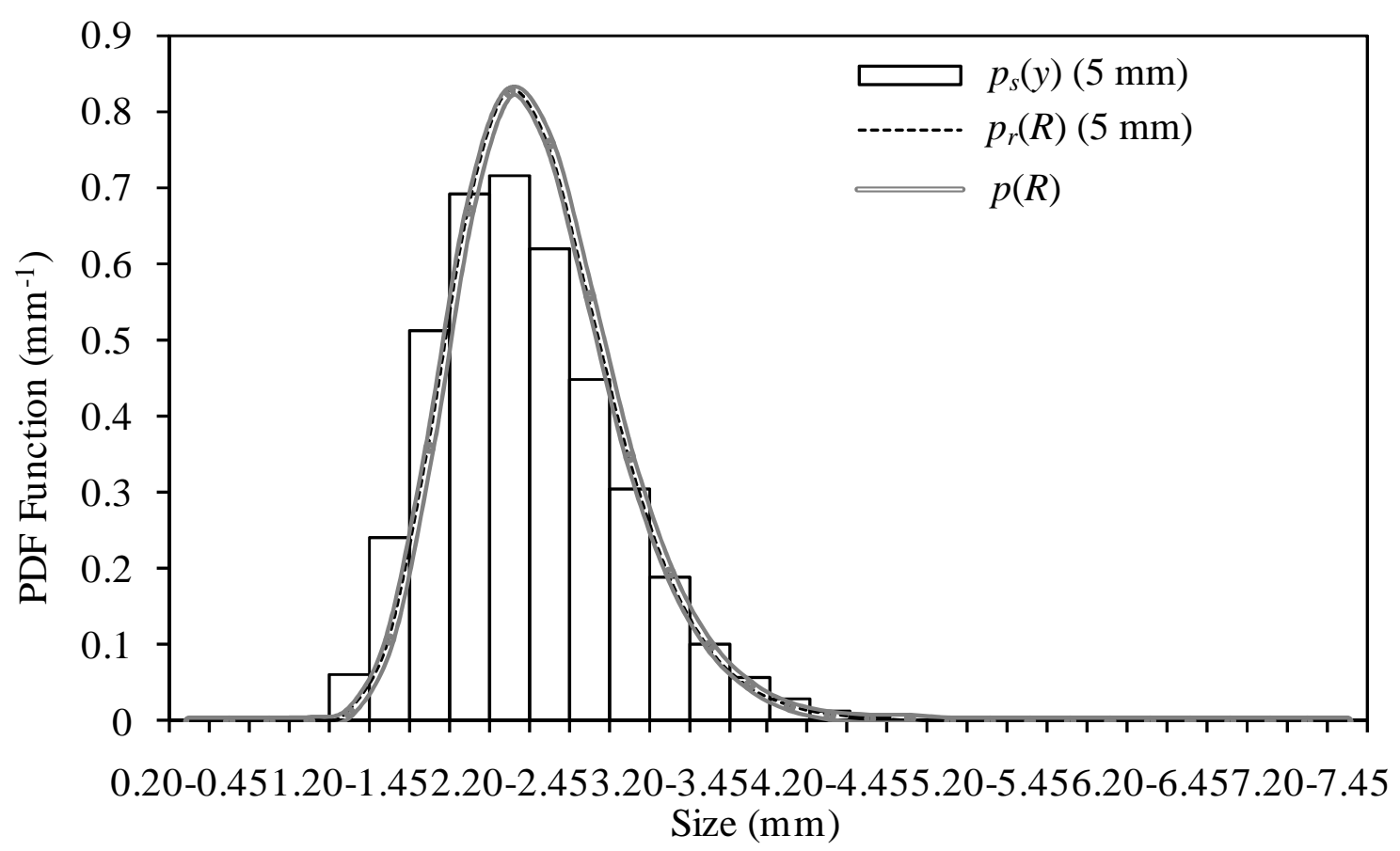

(a)

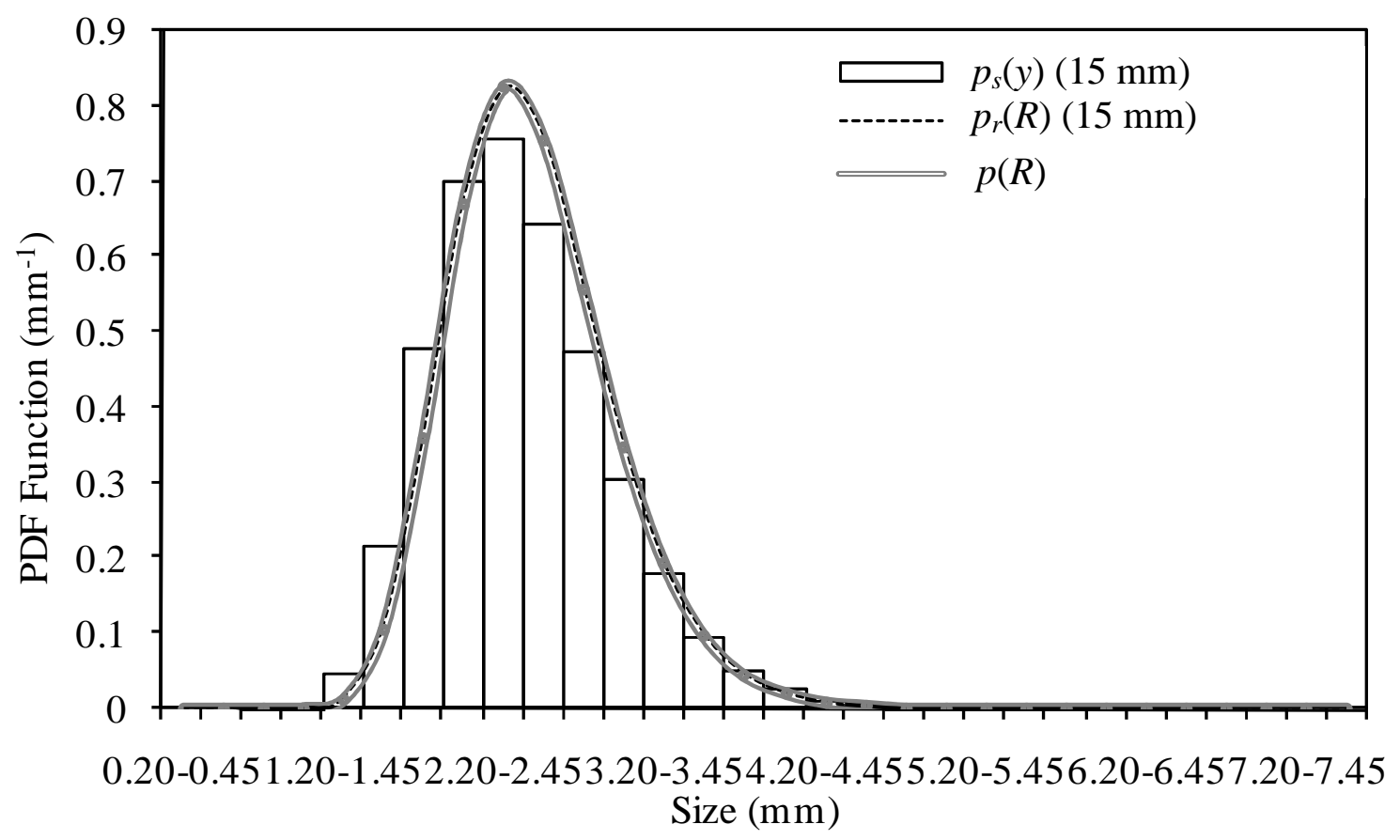

(b)

Figure 14. Evaluation of the effect of different focus adjustment on the stability of the proposed method when the degree of change in magnification is low, (a) focusing distance = $5 \mathrm{~mm}$ from borescope, (b) focusing distance $=15 \mathrm{~mm}$. 


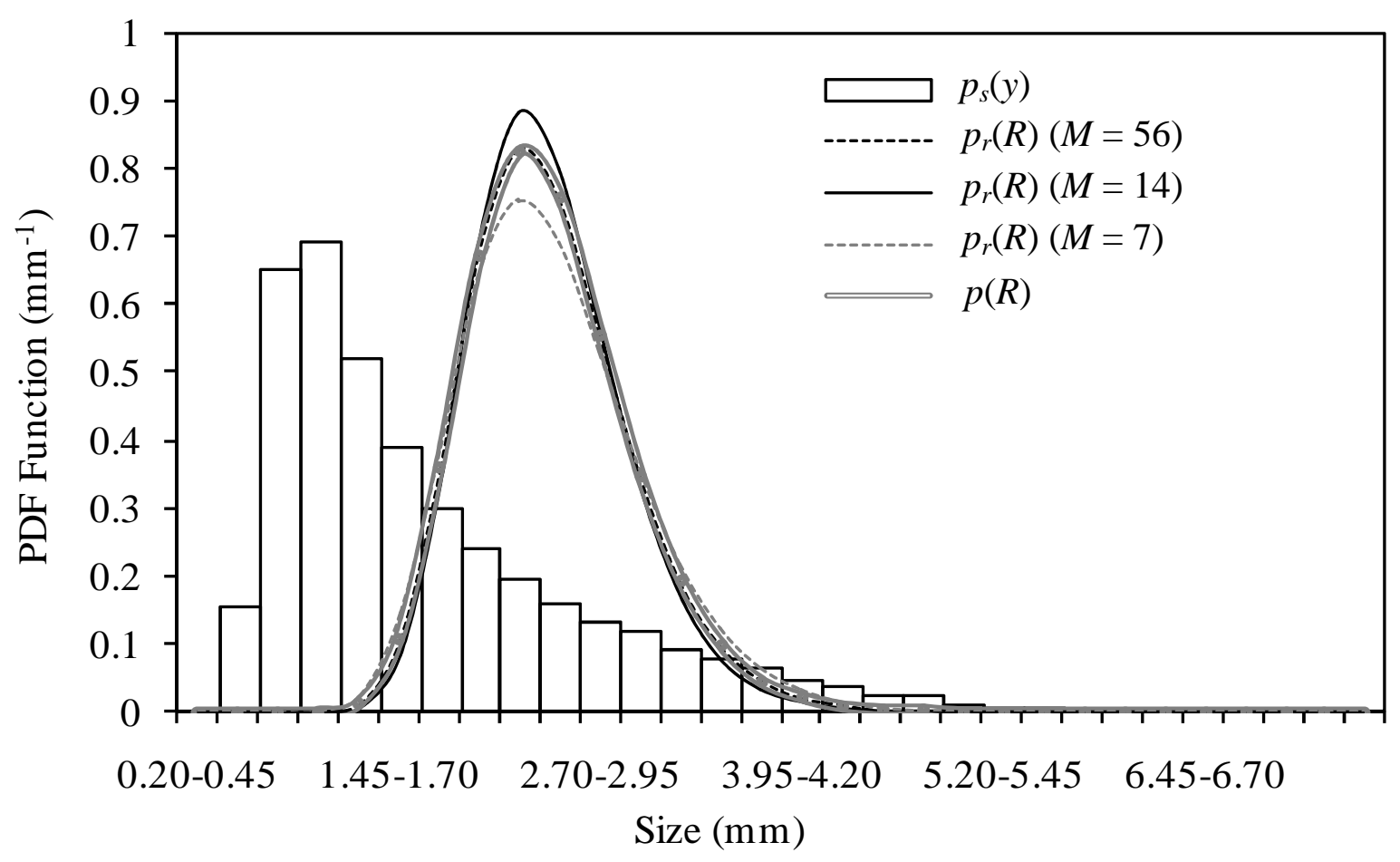

Figure 15. Effect of the number of bins on the stability of the proposed method.

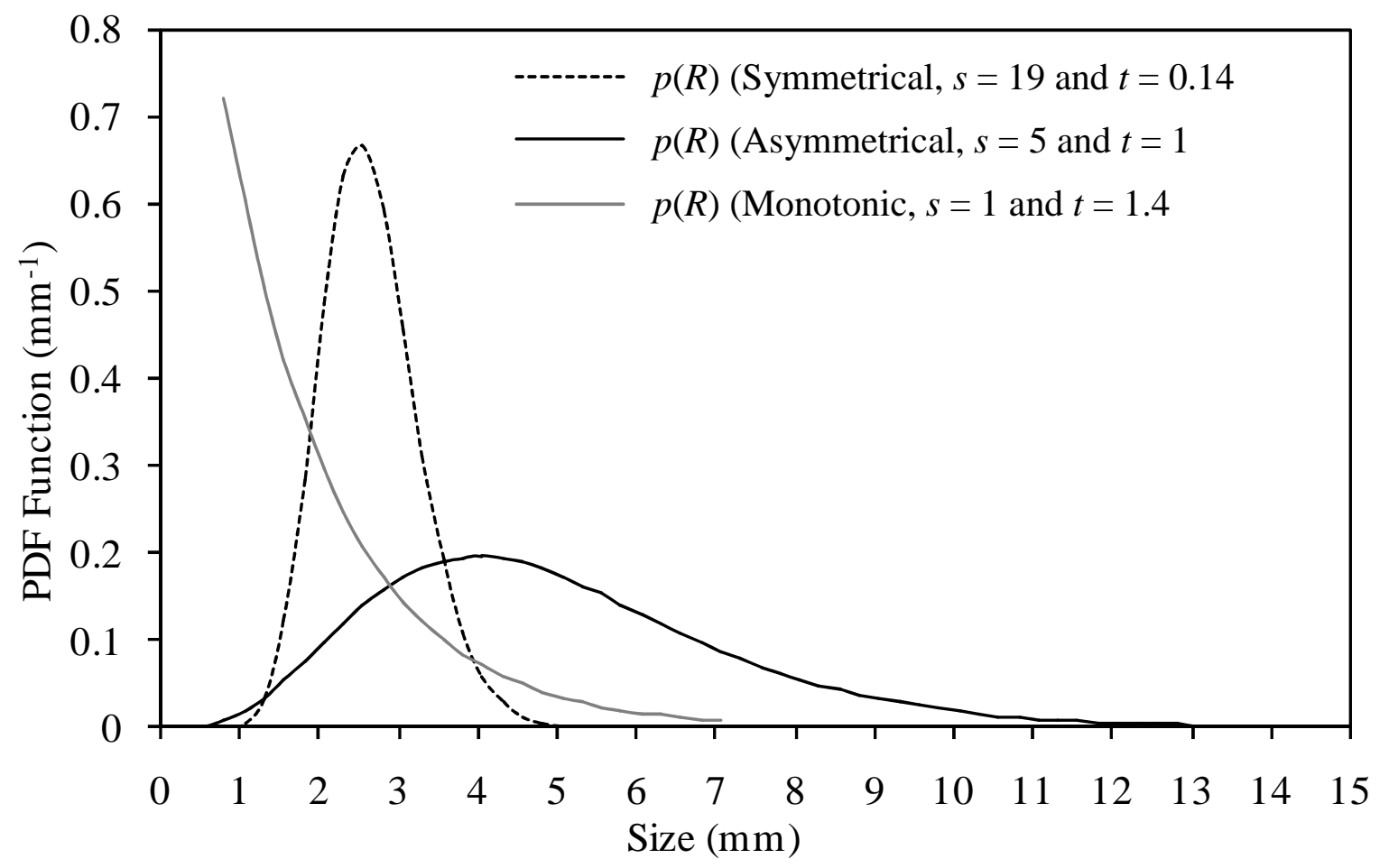

Figure 16. Gamma distribution functions with different shapes. 


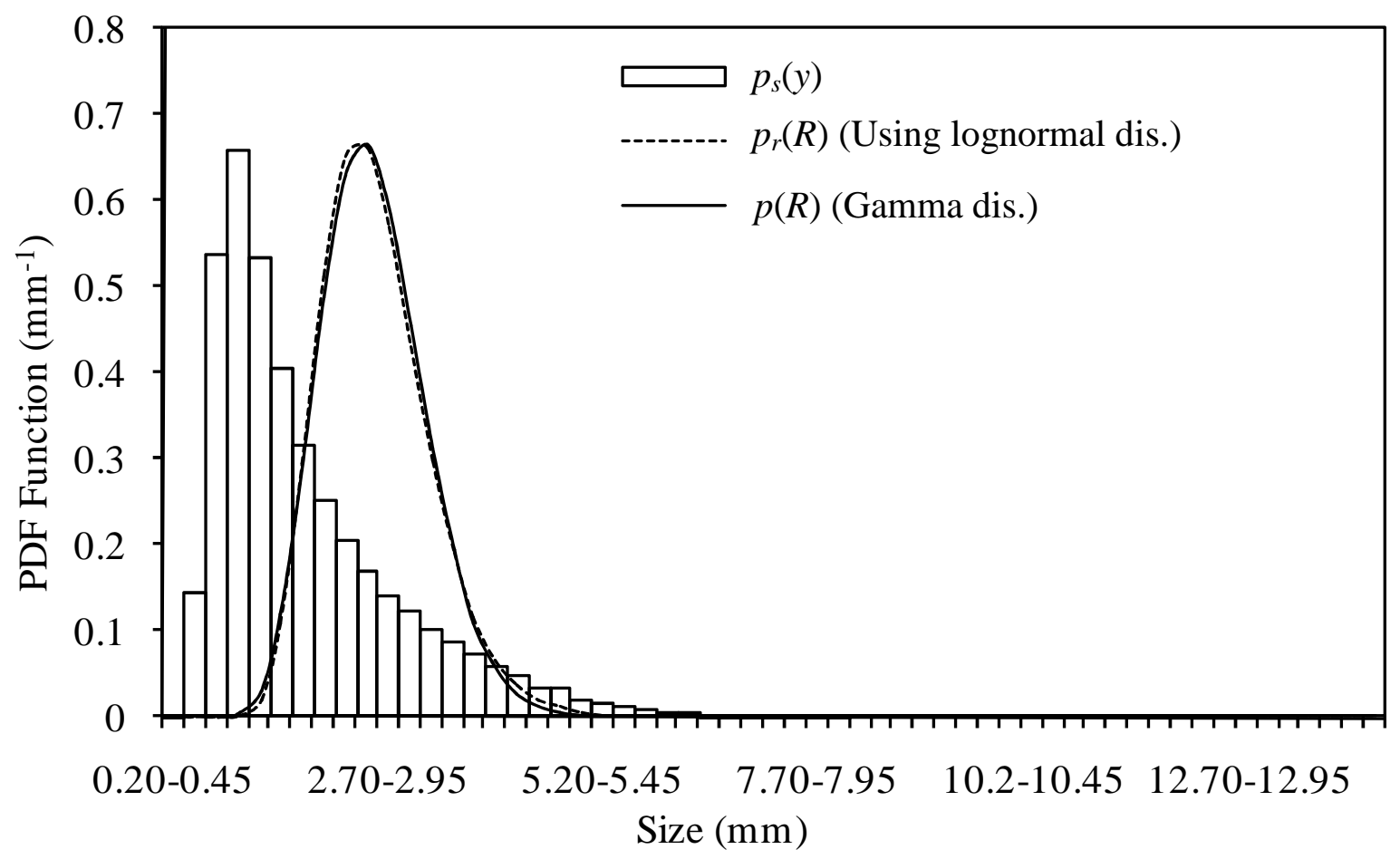

Figure 17. Error analysis for a symmetrical gamma distribution function.

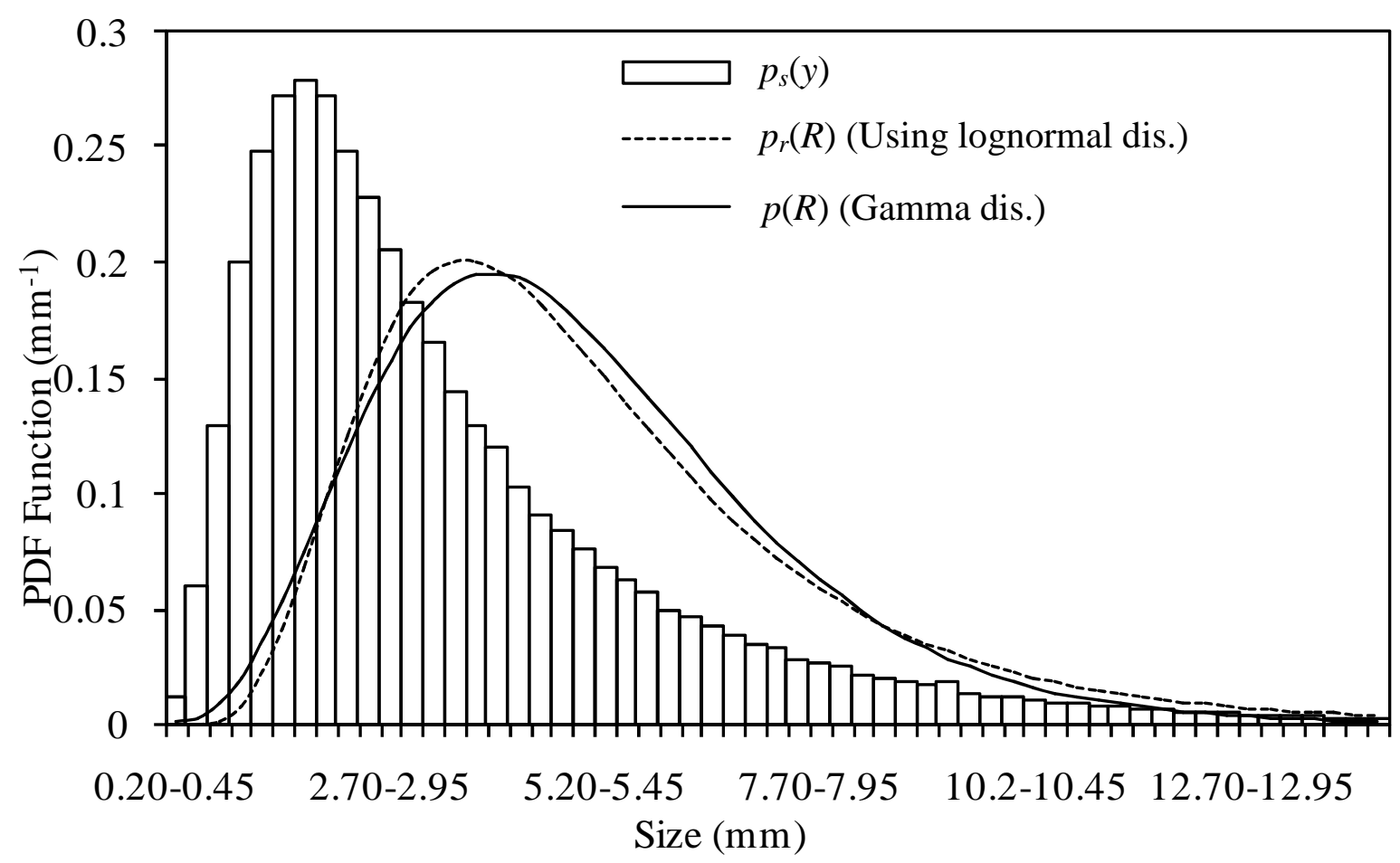

Figure 18. Error analysis for an asymmetrical gamma distribution function. 


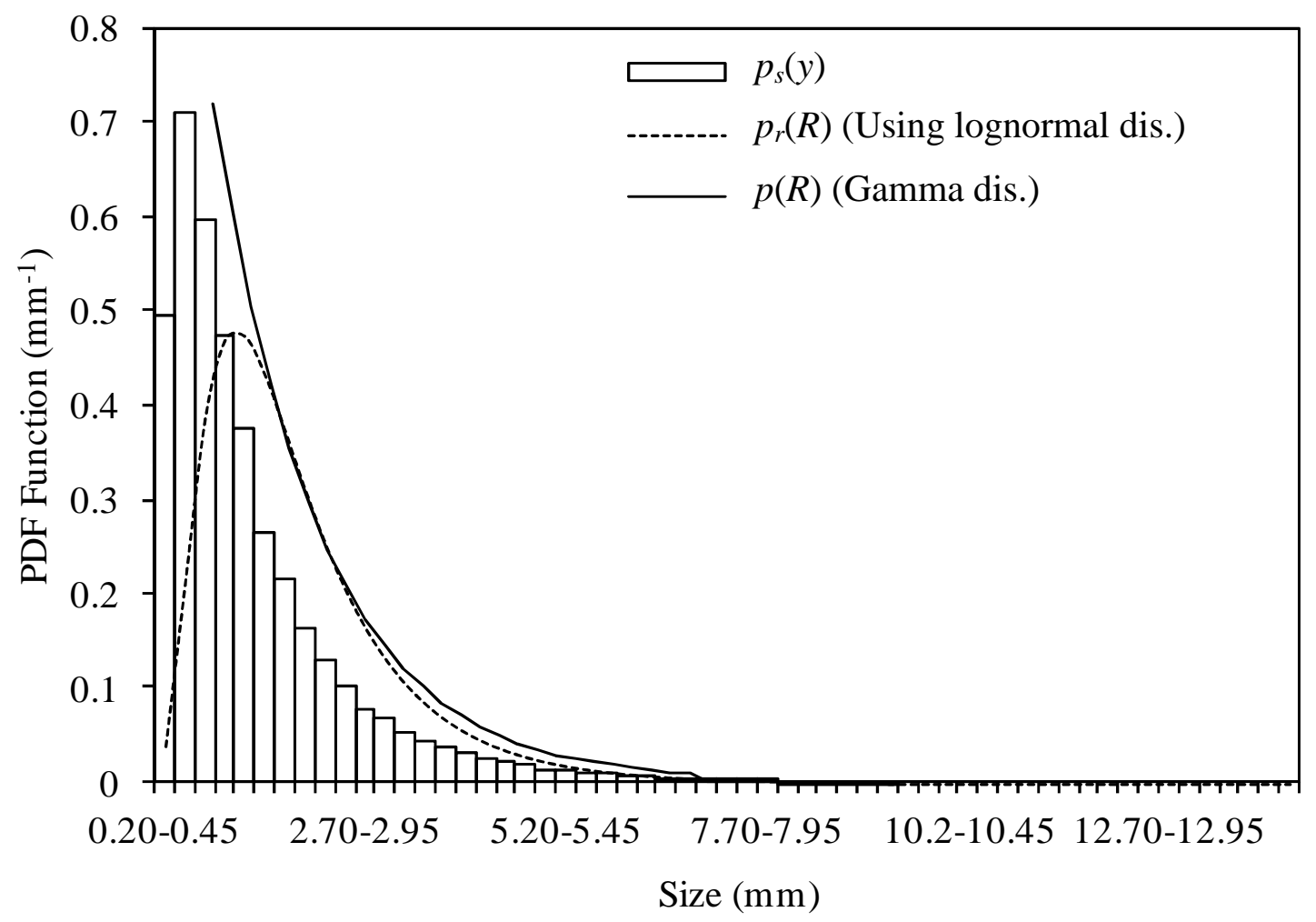

Figure 19. Error analysis for a monotonic gamma distribution function. 